\title{
Convergence and divergence of formal CR mappings
}

\author{
BERNHARD LAMEL \\ Universität Wien \\ Vienna, Austria
}

by

\section{Introduction}

A formal holomorphic map $H:(M, p) \rightarrow M^{\prime}$ from a germ of a real-analytic submanifold $M \subset \mathbb{C}^{N}$ at $p \in M$ into a real-analytic subset $M^{\prime} \subset \mathbb{C}^{N^{\prime}}$ is an $N^{\prime}$-tuple of formal holomorphic power series $H=\left(H_{1}, \ldots, H_{N^{\prime}}\right)$ satisfying $H(p) \in M^{\prime}$ with the property that, for any germ of a real-analytic function $\delta(w, \bar{w})$ at $H(p) \in \mathbb{C}^{N^{\prime}}$ which vanishes on $M^{\prime}$, the formal power series $\delta(H(z), \overline{H(z)})$ vanishes on $M$. There is an abundance of examples showing that formal maps may diverge: After the trivial example of self-maps of a complex submanifold, possibly the simplest non-trivial example is given by the formal maps of $(\mathbb{R}, 0)$ into $\mathbb{R}$ which are just given by the formal power series in $z \in \mathbb{C}$ with real coefficients, that is, by elements of $\mathbb{R}[[z]]$.

It is a surprising fact at first that, for formal mappings between real submanifolds in complex spaces, if one assumes that the trivial examples above are excluded in a suitable sense, the situation is fundamentally different. The first result of this kind was encountered by Chern and Moser in [CM], where - as a byproduct of the convergence of their normal form - it follows that every formal holomorphic invertible map between Levinon-degenerate hypersurfaces in $\mathbb{C}^{N}$ necessarily converges. The convergence problem, that is, deciding whether formal maps, as described above, are in fact convergent, has been studied intensively in different contexts, both for CR manifolds and for manifolds with CR singularities, for which we refer the reader to the papers [Rot], [MMZ2], [LM1], [HY1], [HY2], [HY3], [Sto], [GS] and the references therein. Solutions to the convergence problem have important applications, for example, to the biholomorphic equivalence

The authors were partially supported by the Qatar National Research Fund, NPRP project 7-5111-098. The first author was also supported by the Austrian Science Fund FWF, Project I1776. 
problem, where they yield the fact that formal invertible maps are automatically local biholomorphisms.

The convergence problem for formal CR maps, i.e. when the source manifold $M$ is CR, which this paper mainly focuses on, has received considerable attention. Building on extensive work by Baouendi, Ebenfelt and Rothschild in the 1990s (see [BER1], [BER2], [BER3]), Baouendi, Rothschild and the second author [BMR] proved convergence of formal invertible CR maps under optimal geometric conditions on CR submanifolds $M, M^{\prime} \subset \mathbb{C}^{N}$ : they are the generic submanifolds of $\mathbb{C}^{N}$ that are of finite type and holomorphically non-degenerate. Let us recall that $M$ is holomorphically non-degenerate (in the sense of Stanton [Sta]) if any (locally defined) holomorphic vector field $X$ tangent to an open piece of $M$ must be trivial, and that $M$ is of finite type (in the sense of Bloom-Graham [BG]) if the evaluations at every point $p \in M$ of vector fields in the Lie algebra generated by the $\mathrm{CR}$ and anti-CR vector fields constitute the full complexified tangent space $\mathbb{C} T_{p} M$.

Understanding the convergence problem in the more general case of arbitrary maps between CR submanifolds in complex spaces of arbitrary dimensions $N$ and $N^{\prime}$ has remained much more challenging. The first general convergence result along these lines has only recently been obtained by the authors in [LM1] in the case when $M^{\prime}$ is a strictly pseudoconvex CR manifold; we refer to that paper for an up-to-date account of previous work in that area. In the present paper we introduce a quite different approach to deal with the more general case where we do not assume any strict and, not even any weak, a-priori curvature conditions on $M^{\prime}$.

Let us first discuss the question of how formal CR maps from a given germ $(M, p)$ into $M^{\prime}$ may diverge. It turns out that the natural geometric obstruction to convergence comes from the existence of complex-analytic subvarieties (of positive dimension) lying in $M^{\prime}$. Indeed, assume that $A: \Delta=\{\zeta \in \mathbb{C}:|\zeta|<1\} \rightarrow M^{\prime}$ is a holomorphic disc in $M^{\prime}$. Then, every formal holomorphic power series $f:\left(\mathbb{C}^{N}, p\right) \rightarrow(\mathbb{C}, 0)$ defines a formal $\mathrm{CR}$ map $A \circ f:(M, p) \rightarrow M^{\prime}$. If $f$ is furthermore divergent, so is $A \circ f$. Hence, from this simple observation, one may generate, due to the presence of holomorphic discs in $M^{\prime}$, divergent maps whose image, furthermore, is entirely contained in the set formed by the union of complex-analytic subvarieties (of positive dimension) contained in $M^{\prime}$. Our first main result, Theorem 1.1 below, provides a converse to this by showing that, for any generic submanifold of finite type $M$, the only way to get divergent formal CR maps is by having them being valued in the above mentioned set. Some care is needed in order to define the notion of the image being contained in that set, as the map $H$ is a priori only formal.

For this, we need to recall the definition of points of infinite D'Angelo type (introduced by D'Angelo in [D1] in the context of smooth hypersurfaces) in the real-analytic 
set $M^{\prime}$. A point $p^{\prime} \in M^{\prime}$ is of infinite $D^{\prime}$ Angelo type if there exists a (non-constant) holomorphic disc $A$ : $\Delta \rightarrow M^{\prime}$ with $A(0)=p^{\prime}$. We note that D'Angelo discusses, e.g. in his book [D3], effective ways of deciding whether a given point $p^{\prime}$ is of finite or infinite D'Angelo type. We denote by $\mathcal{E}_{M^{\prime}}$ the collection of all points $p^{\prime} \in M^{\prime}$ which are of infinite D'Angelo type; this, by [D2] and [D3], is a closed subset of $M^{\prime}$. We further say that a formal holomorphic map $H:(M, p) \rightarrow M^{\prime}$ sends $M$ into $\mathcal{E}_{M^{\prime}}$ if there exists a sequence of (germs of) real-analytic maps $h_{k}:\left(\mathbb{C}^{N}, p\right) \rightarrow \mathbb{C}^{N^{\prime}}$ which satisfies $h_{k}=H+O\left(|z-p|^{k+1}\right)$ for $k \in \mathbb{N}$, and such that, for every $k \in \mathbb{N}$, we have $h_{k}((M, p)) \subset \mathcal{E}_{M^{\prime}}$.

We can now state our main theorem.

THEOREM 1.1. Let $M \subset \mathbb{C}^{N}$ be a generic real-analytic submanifold, of finite type at $p \in M$, and $M^{\prime} \subset \mathbb{C}^{N^{\prime}}$ be a real-analytic set, where $N, N^{\prime} \geqslant 2$. For any formal holomorphic mapping $H:(M, p) \rightarrow M^{\prime}$, if $H$ does not send $M$ into $\mathcal{E}_{M^{\prime}}$, then $H$ is convergent.

Theorem 1.1 contains as a special case the main theorem in the aforementioned recent paper of the two authors [LM1], because a strictly pseudoconvex CR manifold $M^{\prime}$ satisfies $\mathcal{E}_{M^{\prime}}=\varnothing$. We also note that, under the additional assumption that $M^{\prime}$ is real algebraic, the conclusion of Theorem 1.1 was obtained by Meylan, Zaitsev and the second author in [MMZ1] and, furthermore, Theorem 1.1 fully settles in the affirmative a question raised in [MMZ2]. Our approach for real-analytic target sets necessarily differs completely from the one taken in the algebraic setting, as all techniques from commutative algebra are not available in this general situation any longer. It turns out that the general setting studied here requires a completely different approach, which we will shortly describe and which provides a new line of attack for a previously untractable problem. Another remarkable feature of Theorem 1.1 we should point out is that it is optimal in the sense that the finite-type assumption on $M$ can not be dropped. Indeed, according to [KS], there exists infinite-type real-analytic hypersurfaces $M$ in $\mathbb{C}^{2}$ through the origin and formal CR divergent self-maps $H:(M, 0) \rightarrow M$ such that $H(M) \not \subset \mathcal{E}_{M}$.

As a particular application of Theorem 1.1, we settle the long-standing question whether the absence of complex-analytic subvarieties in $M^{\prime}$ actually characterizes convergence of every formal map; see [Rot, Conjecture 3.6].

Corollary 1.2. Let $M \subset \mathbb{C}^{N}$ be a generic real-analytic submanifold, of finite type at $p \in M$, and $M^{\prime} \subset \mathbb{C}^{N^{\prime}}$ be a real-analytic set, where $N, N^{\prime} \geqslant 2$. Then, every formal holomorphic mapping $H:(M, p) \rightarrow M^{\prime}$ is convergent if and only if $M^{\prime}$ does not contain any complex-analytic subvariety of positive dimension.

In the equidimensional case $N=N^{\prime}$, some variants of Corollary 1.2 for hypersurfaces have appeared in [BER3] and [Su]. However, even in that special setting, our corollary covers previously unknown cases. The reader will note that Corollary 1.2 is one, among 
others, of the applications of Theorem 1.1. For instance, if $H:(M, p) \rightarrow M^{\prime}$ is a formal $\mathrm{CR}$ map, of generic (complex) rank $\operatorname{Rk} H>1$, and if $\mathcal{E}_{M^{\prime}}$ consists of a single analytic disc passing through $H(p)$, then one can check that $H$ does not send $M$ into $\mathcal{E}_{M^{\prime}}$, and so Theorem 1.1 shows that $H$ must converge. In $\S 2$, we point out a number of other results extending this observation, where we in particular relate conditions on the size of the set of infinite D'Angelo points $\mathcal{E}_{M^{\prime}}$ and the generic rank of $H$ in order to guarantee convergence (see, e.g., Corollary 2.5).

Theorem 1.1 will follow from a far more powerful result, Theorem 2.2, which we state in $\S 2$ below. It gives a general necessary condition for the existence of divergent maps; heuristically speaking, in order for a divergent formal map $H:(M, p) \rightarrow M^{\prime}$ to exist, there must be some integer $1 \leqslant r \leqslant N^{\prime}$ and, for every positive integer $k$, a family of $r$-dimensional complex-analytic subvarieties $\left(\Upsilon_{z}\right)_{z \in M_{k}}$ parameterized by some neighborhood $M_{k}$ of $p$ in $M$, depending in a CR fashion on the parameter $z \in M_{k}$ (in a suitable sense), and such that each submanifold $\Upsilon_{z}$ passes through $H(z)$ and has order of contact at least $k$ with $M^{\prime}$ at $H(z)$. Of course, as $H$ is only a formal mapping, one cannot talk of the point $H(z)$ in $M^{\prime}$ for $z \neq p$, but making this rough intuition precise and exploitable takes up the major part of this paper.

Our approach not only provides restrictions in the sense that for divergent maps to exist the target set $M^{\prime}$ must contain positive-dimensional complex-analytic subvarieties, but also relates the location of these subvarieties with the geometry of $M^{\prime}$ through the map $\left.H\right|_{M}$. This opens the way to study the convergence problem in certain given classes of formal CR mappings (typically, classes that are defined through rank conditions that are stable under small deformations) for which the target set $M^{\prime}$ is allowed to be of infinite D'Angelo type everywhere, i.e. allowing $M^{\prime}=\mathcal{E}_{M^{\prime}}$. We illustrate our claim through three applications fitting the above described setting. We show how the convergence result for formal CR invertible mappings of [BMR] follows as a straightforward consequence of Theorem 2.2 (see Corollary 2.6) and we also completely settle two particularly interesting and previously unknown cases in this paper: optimal results are stated below in $\S 2$ for (transversal) mappings between Levi-non-degenerate hypersurfaces (Corollary 2.7) and mappings with target the tube over the light cone (Corollary 2.8).

The approach developed in the present paper is completely new and radically different from the methods used so far to study the convergence problem for formal CR maps. We therefore believe that the methods described here will be useful in other contexts, and will be adapted for other mapping problems in the future. Indeed, in a forthcoming article, we will use the philosophy of the approach in this paper (together with other ingredients) to study the $\mathcal{C}^{\infty}$-regularity of $\mathrm{CR}$ mappings in complex spaces of different dimension (see [LM2]), in the spirit of the recent work of Berhanu and Xiao [BX1] in the 
case of strictly pseudoconvex targets.

We will now describe our proof in greater detail and also explain the organization of the paper. As already pointed out, we will establish a link between the divergence properties of a formal $\mathrm{CR}$ map and the geometry of the triple $\left(M, M^{\prime}, H\right)$. This is done by means of what we call $k$-approximate formal deformations in $\S 2$. Theorem 2.2 shows that a divergent formal CR mapping which sends a real-analytic generic submanifold $M \subset \mathbb{C}^{N}$ of finite type into some real-analytic set $M^{\prime} \subset \mathbb{C}^{N^{\prime}}$ generates $k$-approximate formal deformations (of the mapping) of any order $k$.

A $k$-approximate formal deformation of the mapping can be viewed as a holomorphic family, depending on a parameter $t \in \mathbb{C}^{r}$, for some $r \geqslant 1$, of formal holomorphic maps $H_{t}:\left(\mathbb{C}^{N}, p\right) \rightarrow \mathbb{C}^{N^{\prime}}$, satisfying $H_{0}=H$, sending $M$ into $M^{\prime}$ up to order $k$ (with respect to the deformation parameter $t$ ) and such that the family $\left(H_{t}\right)_{t \in \mathbb{C}^{r}}$ is an $r$-dimensional deformation of the mapping $H$. We construct these objects, which are crucial for our approach, in $\S 3$ and $\S 4$. The first step in their construction is the introduction and the study of a new invariant attached to $(M, H)$, which we call the divergence rank of $H$. For a generic submanifold $M$ of finite type, the divergence rank provides a suitable measurement of the lack of convergence of the mapping $H$. In order to define the divergence rank, we need to consider the collection of all relations of a certain type satisfied by the mapping $H$, including (but not restricted to) the one coming from the basic mapping identity $H(M) \subset M^{\prime}$. In all previous works on the subject (except notably the algebraic situation studied in [MMZ1]), the basic mapping identity was the only one that was taken into consideration. It appears that this single identity and its prolongations, especially in the case of positive codimension (i.e. $N^{\prime}>N$ ), cannot carry enough information to encode exactly how divergent/convergent the map is.

After we establish the basic properties of the divergence rank in $\S 3.2$, we can identify the right "directions" in which the mapping $H$ can be deformed holomorphically. In $\S 3.3$ we show that such directions can be chosen formally meromorphic. At this point, we should mention that the implementation of the above strategy is based on two useful technical results from our previous work [LM1]. In $\S 4$, we use the formal meromorphic vectors found in $\S 3.3$ to build, for any formal CR map with divergence rank $\geqslant 1$, a formal deformation of the mapping $H$ (with meromorphic coefficients), which is then used to obtain the desired approximate formal deformations of any order, yielding the proof of Theorem 2.2. The proof heavily relies on the well-chosen definition of the divergence rank and on its related properties.

In $\S 5$, from the existence of approximate formal deformations of any order, we derive some more geometric consequences on the existence of families of complex-analytic subvarieties of positive dimension contained in $M^{\prime}$, which yields, among other things, 
the proof of Theorem 1.1. While deriving these geometric consequences (from the given formal approximate statements), we are naturally led to investigate some very interesting open problems related to Artin's approximation theorem [A] in the context of CR geometry (see, e.g., [Mir3]). One relevant question we mention in Conjecture 2.12 concerns an approximation result of strong type in the CR setting. Even though an answer to this question appears presently out of reach in full generality, for the purposes of the present paper, we are able to utilize a parameter version of an approximation result of strong type due to Hickel-Rond [HR]. In the last section of the article, we discuss the notion of $k$-approximate formal deformations for $k=1,2$, and complete the proof of a number of results mentioned in $\S 2$, including the solutions of the convergence problems for formal CR transversal maps between Levi-non-degenerate hypersurfaces and for maps valued in the tube over the light cone.

\section{Existence of divergent formal CR maps: necessary and sufficient conditions}

In this section, we state our most general result (Theorem 2.2), providing a new necessary condition for the existence of divergent formal CR maps. Such a result will be used to derive Theorem 1.1, but we also include here a number of additional convergence results following from Theorem 2.2. Such results cover the case of target real-analytic sets that may be foliated by complex-analytic subvarieties, and hence go beyond the situation treated in Theorem 1.1. We also next discuss in $\S 2.2$ how close to being sufficient the condition obtained in Theorem 2.2 is to guarantee the existence of divergent maps.

\subsection{Approximate formal (holomorphic) deformations}

In what follows, we define the rank of a formal power series map to be the rank of its Jacobian matrix over the quotient field of power series, or, equivalently, the size of a largest non-identically vanishing minor of its Jacobian matrix (see $\S 3.1$ for more details). The following notion will be crucial throughout the paper.

Definition 2.1. Let $M \subset \mathbb{C}^{N}$ be a generic real-analytic submanifold, $M^{\prime} \subset \mathbb{C}^{N^{\prime}}$ be a real-analytic set and $p \in M, N, N^{\prime} \geqslant 2$.

Given a positive integer $k$, a $k$-approximate formal (holomorphic) deformation for $\left(M, M^{\prime}\right)$ at $p$ is a formal holomorphic map $B^{k}:\left(\mathbb{C}_{z}^{N} \times \mathbb{C}_{t}^{r},(p, 0)\right) \rightarrow \mathbb{C}^{N^{\prime}}$ for some integer 
$r \geqslant 1$, with $B^{k}(p, 0) \in M^{\prime}$, satisfying the following conditions:

(i)

$$
\operatorname{Rk} \frac{\partial B^{k}}{\partial t}(z, 0)=r
$$

(ii) for every germ of a real-analytic function $\varrho:\left(M^{\prime}, B^{k}(p, 0)\right) \rightarrow \mathbb{R}$, vanishing on $M^{\prime}$ near $B^{k}(p, 0)$,

$$
\left.\varrho\left(B^{k}(z, t), \overline{B^{k}(z, t)}\right)\right|_{z \in M}=O\left(|t|^{k+1}\right) .
$$

If, in addition, $H:\left(\mathbb{C}^{N}, p\right) \rightarrow \mathbb{C}^{N^{\prime}}$ is a formal holomorphic map sending $M$ into $M^{\prime}$, we say that $H$ admits a $k$-approximate formal deformation if there exists a $k$-approximate formal deformation $B^{k}$ of $\left(M, M^{\prime}\right)$ at $p$ as above satisfying $B^{k}(z, 0)=H(z)$. In that case, we also say that $H$ admits $B^{k}$ as a $k$-approximate formal deformation (of $\left(M, M^{\prime}\right)$ ).

Note that, if $B^{k}$ is a $k$-approximate formal deformation, one may assume without loss of generality that each component of $B^{k}$ belongs to $\mathbb{C}[[z-p]][t]$ (by truncating $B^{k}$ up to order $k$ with respect to $t$ ). Hence $B^{k}$ can be identified with a holomorphic family of formal holomorphic maps $\left(B_{t}^{k}\right)_{t \in \mathbb{C}^{r}}$ where $B_{t}^{k}:=B^{k}(\cdot, t)$ is deforming the map $B_{0}^{k}$.

The main result of the present paper is given by the following theorem, providing a necessary condition for the existence of divergent formal holomorphic maps in terms of approximate formal deformations. We recall that $M$ is said to be of finite type at a point $p \in M$ if the Lie algebra generated by its $\mathrm{CR}$ vector fields and its conjugates spans the full complexified tangent space at $p$ (see, e.g., [BER3] and $[\mathrm{BCH}]$ ).

TheOREM 2.2. Let $M \subset \mathbb{C}^{N}$ be a generic real-analytic submanifold, of finite type at $p \in M$, with $N, N^{\prime} \geqslant 2$. If $H:\left(\mathbb{C}^{N}, p\right) \rightarrow \mathbb{C}^{N^{\prime}}$ is a divergent formal holomorphic map, there exist an integer $r \in\left\{1, \ldots, N^{\prime}\right\}$ and, for every $k \in \mathbb{N}$, a formal holomorphic map

$$
B^{k}:\left(\mathbb{C}^{N} \times \mathbb{C}^{r},(p, 0)\right) \longrightarrow \mathbb{C}^{N^{\prime}}
$$

such that, for every real-analytic set $M^{\prime} \subset \mathbb{C}^{N^{\prime}}$ passing through $H(p)$, if $H(M) \subset M^{\prime}$ then $H$ admits $B^{k}$ as a $k$-approximate formal deformation of $\left(M, M^{\prime}\right)$.

Theorem 2.2 shows that the existence of $k$-approximate formal deformations, for every integer $k$, is a necessary condition for the existence of divergent formal holomorphic maps. It is a natural question if this condition, or an analog, can also serve as a sufficient condition. We will turn to this question in $\S 2.2$ below. Let us emphasize that the $k$ approximate deformations we will construct are universal in the sense that they only depend on the map $H$ and not on the particular real-analytic set $M^{\prime}$ containing $H(M)$.

We see from Theorem 2.2 that, in order to understand the existence of divergent maps, it is essential to understand how the geometry of the triple $\left(M, M^{\prime}, H\right)$ is related to 
the existence/non-existence of approximate formal deformations. To this end, for every positive integer $d$, we denote by $\widetilde{\mathcal{E}}_{M^{\prime}}^{d}$ the set of points in $M^{\prime}$ through which there passes a complex submanifold of dimension $d$. We can use this set to formulate the following geometric consequence of Theorem 2.2.

Corollary 2.3. Let $M \subset \mathbb{C}^{N}$ be a generic real-analytic submanifold, of finite type at $p \in M$, and $M^{\prime} \subset \mathbb{C}^{N^{\prime}}$ be a real-analytic set, with $N, N^{\prime} \geqslant 2$. If $H:(M, p) \rightarrow M^{\prime}$ is a divergent formal holomorphic map, then there exist an integer $r \in\left\{1, \ldots, N^{\prime}\right\}$ and, for any positive integer $k$, a neighborhood $U_{k}$ of $p$ in $\mathbb{C}^{N}$ and a real-analytic map $h_{k}: U_{k} \rightarrow \mathbb{C}^{N^{\prime}}$ such that the following conditions are satisfied:

(a) $h_{k}\left(M \cap U_{k}\right) \subset M^{\prime}$, and $h_{k}$ agrees with $H$ at $p$ up to order $k$;

(b) there exists a Zariski open subset $\Omega_{k}$ of $M \cap U_{k}$ such that $h_{k}\left(\Omega_{k}\right) \subset \tilde{\mathcal{E}}_{M^{\prime}}^{r}$.

In particular, we have that $h_{k}\left(M \cap U_{k}\right) \subset \mathcal{E}_{M^{\prime}}$ for every positive integer $k$.

This result immediately implies Theorem 1.1 mentioned in the introduction. We would like to mention the following geometric consequence of Corollary 2.3. In this corollary, $\left.\operatorname{Rk} H\right|_{M}$ denotes the generic (real) rank of $H$ as a formal map $M \rightarrow \mathbb{C}^{N^{\prime}} \simeq \mathbb{R}^{2 N^{\prime}}$.

Corollary 2.4. Let $M \subset \mathbb{C}^{N}$ be a generic real-analytic submanifold, of finite type at $p \in M$, and $M^{\prime} \subset \mathbb{C}^{N^{\prime}}$ be a real-analytic set, with $N, N^{\prime} \geqslant 2$. If $H:(M, p) \rightarrow M^{\prime}$ is a divergent formal holomorphic map, then there exist an integer $r \in\left\{1, \ldots, N^{\prime}\right\}$ and, in any neighbourhood $U$ of $H(p)$ in $\mathbb{C}^{N^{\prime}}$, a family of $r$-dimensional complex submanifolds $\left(Y_{q}\right)_{q \in X}$ parameterized by a real-analytic submanifold $X \subset U \cap M^{\prime}$ such that $q \in Y_{q} \subset M^{\prime}$ for every $q \in X$, and with $\operatorname{dim}_{\mathbb{R}} X \geqslant\left.\operatorname{Rk} H\right|_{M}$.

A straightforward but noteworthy consequence of Corollary 2.4 providing an easy criterion for convergence of formal holomorphic maps is given by the following.

Corollary 2.5. Let $M \subset \mathbb{C}^{N}$ be a generic real-analytic submanifold, of finite type at $p \in M$, and $M^{\prime} \subset \mathbb{C}^{N^{\prime}}$ be a real-analytic set, with $N, N^{\prime} \geqslant 2$. Denote by $\varkappa_{0}$ the maximum dimension of real-analytic submanifolds contained in $\mathcal{E}_{M^{\prime}}$. Then, any formal holomorphic map $H:(M, p) \rightarrow M^{\prime}$ with $\left.\mathrm{Rk} H\right|_{M}>\varkappa_{0}$ is convergent.

Corollaries 2.3 and 2.5 allow us to conclude the convergence of formal CR maps when their "formal image" is not entirely contained in the set $\mathcal{E}_{M^{\prime}}$ of infinite D'Angelo type points in $M^{\prime}$, or when $\mathcal{E}_{M^{\prime}}$ is not too large. But when $M^{\prime}$ is itself entirely foliated by complex-analytic varieties (which can happen for example if $M^{\prime}$ is a Levi-non-degenerate hyperquadric of positive signature or any homogeneous Levi-degenerate hypersurface; see, e.g., [Fre]), these corollaries are not applicable. However, we shall show that our main result on approximate formal deformations (Theorem 2.2) provides effective results even 
in such cases. We start with a general statement about the existence of 1-approximate deformations.

Given a triple $\left(M, M^{\prime}, H\right)$ as in Theorem 2.2, a 1-approximate formal deformation of $H$ corresponds to the existence of a non-zero formal holomorphic vector field tangent to $M^{\prime}$ along $H(M)$ (see Proposition 6.1). If we furthermore assume that $N=N^{\prime}$ and $H$ is of rank $N$, the existence of a 1-approximate formal deformation of $H$ happens to be equivalent to $M$ being holomorphically degenerate at $p$ in the sense of [Sta] (see Proposition 6.2). Hence, Theorem 2.2 implies in this specific setting the well-known following result of $[\mathrm{BMR}]$ and $[\mathrm{Su}]$.

Corollary 2.6. Let $M, M^{\prime} \subset \mathbb{C}^{N}$ be (connected) generic real-analytic submanifolds and $p \in M, N \geqslant 2$. If $M$ is of finite type at $p$ and $H:\left(\mathbb{C}^{N}, p\right) \rightarrow \mathbb{C}^{N^{\prime}}$ is a divergent formal holomorphic map of rank $N$ with $H(M) \subset M^{\prime}$, then $M$ is holomorphically degenerate.

Regarding 2-approximate formal deformations, it is pretty easy to see that there are no such objects when $M^{\prime}$ is a strongly pseudoconvex CR manifold (see Proposition 6.3). On the other hand, the situation is different and, in some sense, more interesting when we study the existence of 2-approximate formal deformations for CR transversal formal maps between Levi-non-degenerate hypersurfaces. Let us recall that, if $M$ is Levi-nondegenerate and connected, then the minimum (resp. maximum) of the numbers of positive and negative eigenvalues of its Levi form is the same at each point of $M$ and is called the signature (resp. cosignature) of $M$. Let us also recall that, if $M$ and $M^{\prime}$ are two realanalytic hypersurfaces in $\mathbb{C}^{N}$ and $\mathbb{C}^{N^{\prime}}$, respectively, with $p \in M$, a formal holomorphic map $H:\left(\mathbb{C}^{N}, p\right) \rightarrow \mathbb{C}^{N^{\prime}}$ sending $M$ into $M^{\prime}$ is called $C R$ transversal (at $p$ ) if

$$
T_{H(p)}^{1,0} M^{\prime}+d H\left(T_{p}^{1,0}\left(\mathbb{C}^{N}\right)\right)=T_{H(p)}^{1,0} \mathbb{C}^{N^{\prime}}
$$

The following result is an application of Theorem 2.2 in the context of transversal maps between Levi-non-degenerate hypersurfaces.

Corollary 2.7. Let $M \subset \mathbb{C}^{N}$ and $M^{\prime} \subset \mathbb{C}^{N^{\prime}}$ be (connected) real-analytic Levi-nondegenerate hypersurfaces, of signature $\ell$ and $\ell^{\prime}$, respectively, with $N, N^{\prime} \geqslant 2$. Assume that $M$ and $M^{\prime}$ have either the same signature (i.e. $\left.\ell=\ell^{\prime}\right)$ or cosignature (i.e. $N-\ell=N^{\prime}-\ell^{\prime}$ ). Then, for every $p \in M$, if $H:(M, p) \rightarrow M^{\prime}$ is a formal holomorphic map which is $C R$ transversal at $p$, then $H$ is convergent.

More specifically, this result will follow from Theorem 2.2 by showing that, under the above conditions $\ell=\ell^{\prime}$ or $\ell^{\prime}-\ell=N^{\prime}-N$, a CR transversal formal holomorphic map $H$ does not admit any 2-approximate formal deformation (see Proposition 6.4). Corollary 2.7 is optimal in the sense that, if the condition on the signatures of $M$ and $M^{\prime}$ is violated, then 
there exists examples of Levi-non-degenerate hyperquadrics and CR transversal formal holomorphic maps which are divergent. This is explained in detail in Remark 6.6.

In the case $\ell=\ell^{\prime}=0$, Corollary 2.7 is a special case of the strongly pseudoconvex setting already settled in [LM1] (or contained in Corollary 1.2), in the sense that, in this setting, the transversality assumption is superfluous. Besides that case, Corollary 2.7 is a completely new result, which has not appeared even in special cases in the literature. It is worth pointing out the similarity between the condition on the signatures in our Corollary 2.7 and the one appearing in the work of Baouendi-Huang $[\mathrm{BH}]$ in their study of the rigidity of holomorphic maps between hyperquadrics. We also note that, if $\ell=0$, that is, if the source hypersurface $M$ is strongly pseudoconvex, then Corollary 2.7 not only guarantees the convergence of all formal CR transversal maps in the case that $M^{\prime}$ is strictly pseudoconvex, but also under the condition $\ell^{\prime}=N^{\prime}-N$. This condition is analogous to the one given in the works by Berhanu-Xiao and the second author [BX2], [Mir4] dealing with the smoothness/analyticity of CR maps.

We should also add that if the codimension is 1 , that is, $N^{\prime}=N+1$, the condition of Corollary 2.7 on the signatures of $M$ and $M^{\prime}$ is always satisfied. We thus also recover a result of the second author [Mir2, Theorem 1.2] (see also [L] for an earlier related result).

We conclude with a last application of Theorem 2.2 (not accessible from Corollary 2.3) that allows to treat formal maps valued in the tube over the light cone. Recall that the latter is the regular part of the real-algebraic variety given by

$$
\mathbb{T}^{N^{\prime}}:=\left\{\left(z_{1}, \ldots, z_{N^{\prime}}\right) \in \mathbb{C}^{N^{\prime}}:\left(\operatorname{Re} z_{N^{\prime}}\right)^{2}=\sum_{j=1}^{N^{\prime}-1}\left(\operatorname{Re} z_{j}\right)^{2}\right\} .
$$

As is well known, the regular points of $\mathbb{T}^{N^{\prime}}$ constitute a holomorphically non-degenerate real hypersurface foliated by complex lines. For any real-analytic generic submanifold $M$ of finite type in $\mathbb{C}^{N}$ and for $M^{\prime}=\mathbb{T}^{N^{\prime}}$, we shall determine in Proposition 6.8 all formal CR maps sending $M$ into $\mathbb{T}^{N^{\prime}}$ admitting a 2-approximate formal deformation. In conjunction with Theorem 2.2, this description immediately yields the following result.

COROLlary 2.8. Let $M \subset \mathbb{C}^{N}$ be a generic real-analytic submanifold in $\mathbb{C}^{N}$, of finite type at $p \in M$, and $\mathbb{T}^{N^{\prime}} \subset \mathbb{C}^{N^{\prime}}$ be the tube over the light cone, with $N, N^{\prime} \geqslant 2$. Then, every formal holomorphic map $H:(M, p) \longrightarrow \mathbb{T}^{N^{\prime}}$ with $\mathrm{Rk} H \geqslant 2$ is convergent.

We note that the rank condition in Corollary 2.8 is again optimal, in the sense that there exist divergent formal holomorphic maps of rank 1. Indeed, if $\eta(z)$ is any divergent formal holomorphic function, the map $z \mapsto(\eta(z), 0, \ldots, 0, \eta(z))$ is an example of a divergent formal holomorphic map valued in $\mathbb{T}^{N^{\prime}}$. Actually, all of the possible divergent maps can be completely characterized (see Corollary 6.9). 


\subsection{Approximate formal deformations vs. holomorphic deformations}

Theorem 2.2 shows that the existence of divergent formal CR maps imply the existence of $k$-approximate formal deformations for every integer $k$. We shall now explain to what extent the existence of approximate formal deformations is relevant in order to establish the existence of divergent formal CR maps. To this end, following the lines developed by the second author for the study of the analyticity problem for CR maps in [Mir4], we first introduce the notion of holomorphic deformation as follows.

Definition 2.9. Let $M \subset \mathbb{C}^{N}$ be a generic real-analytic submanifold and $M^{\prime} \subset \mathbb{C}^{N^{\prime}}$ be a real-analytic set and $p \in M$, with $N, N^{\prime} \geqslant 2$. A holomorphic deformation for $\left(M, M^{\prime}\right)$ at $p$ is a (germ of a) holomorphic map $B:\left(\mathbb{C}_{z}^{N} \times \mathbb{C}_{t}^{r},(p, 0)\right) \rightarrow \mathbb{C}^{N^{\prime}}$ for some integer $r \geqslant 1$ satisfying the following conditions:

(i)

$$
\operatorname{Rk} \frac{\partial B}{\partial t}(z, t)=r
$$

(ii) $B\left(M \times \mathbb{C}^{r}\right) \subset M^{\prime}$.

If, in addition, $H:\left(\mathbb{C}^{N}, p\right) \rightarrow \mathbb{C}^{N^{\prime}}$ is a formal holomorphic map with $H(M) \subset M^{\prime}$, we say that $B$ is a holomorphic deformation of $H$ (or that $H$ admits $B$ as a holomorphic deformation) if there exists a formal power series map $G:\left(\mathbb{C}^{N}, p\right) \rightarrow\left(\mathbb{C}^{r}, 0\right)$ such that $H(z)=B(z, G(z))$.

Given a holomorphic deformation $B$ for $\left(M, M^{\prime}\right)$ at $p$ as in Definition 2.9, one may also define the rank of the deformation to be

$$
\operatorname{Rk} \frac{\partial B}{\partial z}(z, t)
$$

We conjecture the following necessary and sufficient condition for the convergence of formal CR maps of a prescribed rank in terms of the non-existence of holomorphic deformations. 
Conjecture 2.10. Let $M \subset \mathbb{C}^{N}$ be a generic real-analytic submanifold, of finite type at $p \in M$, and $M^{\prime} \subset \mathbb{C}^{N^{\prime}}$ be a real-analytic set, with $N, N^{\prime} \geqslant 2$. Then for every positive integer $\varkappa$, the following conditions are equivalent:

(i) any formal holomorphic map $H:\left(\mathbb{C}^{N}, p\right) \rightarrow \mathbb{C}^{N^{\prime}}$ sending $M$ into $M^{\prime}$ with $\mathrm{Rk} H \geqslant \varkappa$ is convergent;

(ii) there does not exist any holomorphic deformation for $\left(M, M^{\prime}\right)$ at $p$ of rank at least $\varkappa$.

The implication (i) $\Rightarrow$ (ii) can be easily obtained as follows. Suppose that there exists a holomorphic deformation $B$ of $\left(M, M^{\prime}\right)$ at $p$ of rank $\geqslant \varkappa$. Then, for any divergent formal power series $\varpi(z) \in \mathbb{C}[[z-p]]$ vanishing at $p$, for $u \in \mathbb{C}^{*}$ and for $\delta_{1}, \ldots, \delta_{r} \in \mathbb{C}$ sufficiently small generic values, the formal map $z \mapsto B\left(z, u \varpi(z)+\delta_{1}, \delta_{2}, \ldots, \delta_{r}\right)$ is divergent. Indeed, for $\delta_{1}, \ldots, \delta_{r} \in \mathbb{C}$ sufficiently small generic values and $u \in \mathbb{C}$, we have

$$
\operatorname{Rk} \frac{\partial B}{\partial t}\left(z, u \varpi(z)+\delta_{1}, \delta_{2}, \ldots, \delta_{r}\right)=r \quad(\text { in } \mathbb{C}[[z-p]])
$$

and the formal map

$$
z \longmapsto B\left(z, u \varpi(z)+\delta_{1}, \delta_{2}, \ldots, \delta_{r}\right)
$$

has rank $\geqslant \varkappa$. If $B\left(z, u \varpi(z)+\delta_{1}, \delta_{2}, \ldots, \delta_{r}\right)$ were convergent for $u \neq 0$, it would follow that $\varpi(z)$ would be convergent too, as a consequence of e.g. [Mir1, Proposition 4.2]. Hence for the above choices of $u$ and $\delta_{1}, \ldots, \delta_{r}$, the full map (2.2) is therefore divergent, sends $(M, p)$ into $M^{\prime}$ and has rank $\geqslant \varkappa$. Note that in case $\varkappa=1$, condition (ii) is equivalent to say that $M^{\prime}$ does not contain any holomorphic curve, and hence Conjecture 2.10 in this case is true, in view of Corollary 1.2. The converse implication in Conjecture 2.10 for $\varkappa>1$ seems to be still open. However, Theorem 2.2 can be seen as a contribution to its solution. Namely, from Theorem 2.2 we have the following result.

Corollary 2.11. Let $M \subset \mathbb{C}^{N}$ be a generic real-analytic submanifold, of finite type at $p \in M$, and $M^{\prime} \subset \mathbb{C}^{N^{\prime}}$ be a real-analytic set, with $N, N^{\prime} \geqslant 2$. If $H:\left(\mathbb{C}^{N}, p\right) \rightarrow \mathbb{C}^{N^{\prime}}$ is a divergent formal holomorphic map with $H(M) \subset M^{\prime}$, there exists an integer $r \in\left\{1, \ldots, N^{\prime}\right\}$, and for any $k \in \mathbb{N}$, a formal holomorphic map $B^{k}:\left(\mathbb{C}^{N} \times \mathbb{C}^{r},(p, 0)\right) \rightarrow \mathbb{C}^{N^{\prime}}$ such that $B^{k}$ is a k-approximate formal deformation of $H$ of rank at least $\mathrm{Rk} H$.

We believe, in fact, that the existence of $k$-approximate formal deformations for every integer $k$ for a given map $H$ as above happens to be equivalent to the existence of a holomorphic deformation of that map. In fact, we conjecture the following, which in view of Corollary 2.11 implies Conjecture 2.10. 
Conjecture 2.12. Let $M \subset \mathbb{C}^{N}$ be a generic real-analytic submanifold, of finite type at $p \in M$, and $M^{\prime} \subset \mathbb{C}^{N^{\prime}}$ be a real-analytic set, with $N, N^{\prime} \geqslant 2$. Let $H:\left(\mathbb{C}^{N}, p\right) \rightarrow \mathbb{C}^{N^{\prime}}$ be a formal holomorphic map with $H(M) \subset M^{\prime}$. Assume that there exist an integer $r \in$ $\left\{1, \ldots, N^{\prime}\right\}$ and, for any $k \in \mathbb{N}$, a formal holomorphic map $B^{k}:\left(\mathbb{C}^{N} \times \mathbb{C}^{r},(p, 0)\right) \rightarrow \mathbb{C}^{N^{\prime}}$ such that $B^{k}$ is a $k$-approximate formal deformation of $H$. Then, there exists a holomorphic map $B:\left(\mathbb{C}^{N} \times \mathbb{C}^{r},(p, 0)\right) \rightarrow \mathbb{C}^{N^{\prime}}$ that is a holomorphic deformation for $\left(M, M^{\prime}\right)$ at $p$ of rank at least $\operatorname{Rk} H$.

Hence, assuming the validity of Conjecture 2.12, Theorem 2.2 provides a necessary condition that is also sufficient regarding the existence of divergent formal CR maps. Note that results of a similar flavour as that of Conjecture 2.12 can be found in the existing literature (see, e.g., [Z] and [Mir3]), but Conjecture 2.12 involves a much more challenging and general situation than what can so far be found.

\section{Properties of divergent formal maps}

Let $M \subset \mathbb{C}^{N}$ be a generic real-analytic submanifold and $p \in M$. Throughout the paper, $(M, p)$ will denote the germ of $M$ at $p$. In this section, given a formal holomorphic map $H:\left(\mathbb{C}^{N}, p\right) \rightarrow \mathbb{C}^{N^{\prime}}$, we will introduce the first main ingredient for our construction of approximate deformations, namely a new numerical invariant attached to the pair $(M, H)$, which we call the divergence rank of $(M, H)$ (or simply $H$ ). It will then be shown that such an invariant suitably measures, when $M$ is of finite type at $p$, the lack of convergence of the map $H$.

\subsection{Notation and preliminary results}

Throughout the paper, for $k \geqslant 1$ and $t=\left(t_{1}, \ldots, t_{k}\right) \in \mathbb{C}^{k} \simeq \mathbb{R}^{2 k}$, we denote by $\mathbb{C}[[t, \bar{t}]]$ the ring of formal power series, by $\mathbb{C}\{t, \bar{t}\}$ the ring of convergent power series, and by $\mathbb{C}[t, \bar{t}]$ the ring of polynomials in $t$ and $\bar{t}$ with complex coefficients. The subring of holomorphic formal power series (resp. holomorphic convergent power series) is denoted by $\mathbb{C}[[t]]$ (resp. $\mathbb{C}\{t\})$. The field of fractions of $\mathbb{C}[[t, \bar{t}]]$ (resp. $\mathbb{C}[[t]])$ is denoted by $\mathbb{C}((t, \bar{t}))$ (resp. $\mathbb{C}((t))$ ). Note that, by complexification, $\mathbb{C}[[t, \bar{t}]]$ (resp. $\mathbb{C}\{t, \bar{t}\}$ ) may be identified with the ring of formal holomorphic power series $\mathbb{C}[[t, \tau]]$ (resp. holomorphic convergent power series $\mathbb{C}\{t, \tau\})$ in $2 k$ complex variables $(t, \tau)$. For a formal power series $g(t) \in \mathbb{C}[[t]]$, we denote by $\bar{g}(t)$ the formal power series whose coefficients are the complex conjugates of those of $g(t)$. Furthermore, given a positive integer $m$, for a formal power series mapping $h(t, \bar{t}) \in(\mathbb{C}[[t, \bar{t}]])^{m}$, the rank of $h$, denoted by $\mathrm{Rk} h$, is the rank of its Jacobian matrix 
over the field $\mathbb{C}((t, \bar{t}))$; it is equal to the size of a largest non identically vanishing (in $\mathbb{C}[[t, \bar{t}]])$ minor of its Jacobian matrix.

All of the previous notation will also be used for rings of power series over the field of real numbers, as well as for power series that are not necessarily centered at the origin. For instance, $\mathbb{C}[[t-q, \bar{t}-\bar{q}]]$ denotes the ring of convergent power series in $t$ and $\bar{t}$ centered at $q$, with complex coefficients.

Let $M \subset \mathbb{C}_{z}^{N}$ be a real-analytic generic submanifold of codimension $d$ and $p \in M$. Then, there exists a real-analytic vector-valued defining function $\varrho:\left(\mathbb{C}^{N}, p\right) \rightarrow \mathbb{R}^{d}$,

$$
\varrho=\left(\varrho_{1}, \ldots, \varrho_{d}\right) \in(\mathbb{R}\{z-p, \bar{z}-\bar{p}\})^{d},
$$

for $M$ near $p$. This means that $\varrho$ is real-valued, $M=\{\varrho=0\}$ as germs at $p$ and

$$
\partial \varrho_{1} \wedge \ldots \wedge \partial \varrho_{d}(p, \bar{p}) \neq 0
$$

The components $\varrho_{1}, \ldots, \varrho_{d}$ generate an ideal $\mathcal{I}(M) \subset \mathbb{C}\{z-p, \bar{z}-\bar{p}\}$. The ring of germs of real-analytic functions on $M$ at $p$ is then given by

$$
\mathbb{C}\{M\}:=\frac{\mathbb{C}\{z-p, \bar{z}-\bar{p}\}}{\mathcal{I}(M)} .
$$

The ideal of formal power series that vanish on $M$, i.e. $\widehat{\mathcal{I}}(M)=\mathcal{I}(M) \mathbb{C}[[z-p, \bar{z}-\bar{p}]]$ is the ideal of formal power series $S(z, \bar{z}) \in \mathbb{C}[[z-p, \bar{z}-\bar{p}]]$ such that, for any real-analytic (or formal) parametrization $\psi:\left(\mathbb{R}_{x}^{2 N-d}, 0\right) \rightarrow(M, p)$, we have $S(\psi(x), \bar{\psi}(x))=0$. It is easy to check that both $\mathcal{I}(M)$ and $\widehat{\mathcal{I}}(M)$ are prime ideals (in $\mathbb{C}\{z-p, \bar{z}-\bar{p}\}$ and $\mathbb{C}[[z-p, \bar{z}-\bar{p}]]$, respectively). We define the ring of formal power series on $M$ to be the quotient ring

$$
\mathbb{C}[[M]]:=\frac{\mathbb{C}[[z-p, \bar{z}-\bar{p}]]}{\widehat{\mathcal{I}}(M)} .
$$

Note that $\mathbb{C}[[M]]$ is an integral domain, and the closure of $\mathbb{C}\{M\}$ with respect to the topology generated by its maximal ideal. One should in principle indicate in this notation that the ring $\mathbb{C}[[M]]$ depends on the point $p$, but we will omit this, as the point $p$ will be fixed throughout the paper, and therefore identify $M$ with the germ of $M$ at $p$. For any formal power series $A(z, \bar{z}) \in \mathbb{C}[[z-p, \bar{z}-\bar{p}]]$, we will denote by $\left.A(z, \bar{z})\right|_{M}$ (or $\left.\left.A(z, \bar{z})\right|_{z \in M}\right)$ the image of $A$ in $\mathbb{C}[[M]]$ under the canonical projection. The field of fractions of $\mathbb{C}[[M]]$ will be denoted by $\mathbb{C}((M))$.

Let $M$ and $p$ be as above, and $n$ denote the $\mathrm{CR}$ dimension of $M$. For any realanalytic (or formal) $\mathrm{CR}$ vector field $\bar{L}$ tangent to $M$ near $p$, we can define $\bar{L} u \in \mathbb{C}((M))$ for $u \in \mathbb{C}((M))$, since the coefficients of $\bar{L}$ belong to $\mathbb{C}\{z-p, \bar{z}-\bar{p}\}$ (resp. $\mathbb{C}[[z-p, \bar{z}-\bar{p}]]$ ), 
and $\bar{L}$ annihilates $\mathcal{I}(M)$ (resp. $\widehat{\mathcal{I}}(M))$. We say that $u$ is CR if $\bar{L} u=0$ for every such CR vector field $\bar{L}$. It is well known that $u \in \mathbb{C}((M))$ is $\mathrm{CR}$ if and only there exists $\eta \in \mathbb{C}((z))$, where $\mathbb{C}((z))$ denotes the quotient field of $\mathbb{C}[[z]]$ such that $u=\left.\eta\right|_{M}$ (see, e.g., [MMZ1]). Since $\bar{L}$ satisfies the Leibniz rule, the collection of all CR elements in $\mathbb{C}((M))$ is a subfield, which we will denote by $\mathrm{CR}((M))$.

Suppose now that we are given a real-analytic set $M^{\prime} \subset \mathbb{C}^{N^{\prime}}$. We recall that a formal holomorphic mapping $H:\left(\mathbb{C}_{z}^{N}, p\right) \rightarrow \mathbb{C}_{w}^{N^{\prime}}$ sends $M$ into $M^{\prime}$, and write $H(M) \subset M^{\prime}$, or

$$
H:(M, p) \longrightarrow M^{\prime}
$$

if, for every germ of a real-analytic function $A(w, \bar{w}) \in \mathbb{C}\{w-H(p), \bar{w}-\overline{H(p)}\}$ vanishing on $M^{\prime}$ near $H(p)$, the power series $A(H(z), \overline{H(z)}) \in \widehat{\mathcal{I}}(M)$ or, equivalently,

$$
\left.A(H(z), \overline{H(z)})\right|_{M}=0
$$

In case $H$ is convergent, the reader can easily check that this means that the germ of $H$ at $p$ sends the germ of $M$ (at $p$ ) into $M^{\prime}$ in the usual sense.

We now collect a few preliminary but important results from [LM1]. The first one, Lemma 3.1, follows from using similar arguments as those of [LM1, Lemma 4.5], and is very much related to a corresponding statement in the smooth category in [BX1]. We leave the details to the reader.

Lemma 3.1. Let $M \subset \mathbb{C}^{N}$ be a generic real-analytic submanifold, $p \in M$, and $m$ and $r$ be two positive integers with $r<m$. Let $\mathbb{F}$ be a subfield of $\mathbb{C}((M))$, and consider a subspace $\mathcal{G}$ of $(\mathbb{F})^{m}$ over $\mathbb{F}$ of dimension $r$ which is closed under the application of $C R$ vector fields. Then, the $(m-r)$-dimensional subspace $\operatorname{Ann}(\mathcal{G})$ can be generated by $C R$ vectors, i.e. there exist $\mathbb{F}$-linearly independent $V^{1}, \ldots, V^{m-r} \in(\mathrm{CR}((M)))^{m} \cap(\mathbb{F})^{m}$ satisfying

$$
A \cdot V^{\ell}:=\sum_{k=1}^{m} A_{k} V_{k}^{\ell}=0 \text { for all } A \in \mathcal{G}, \quad 1 \leqslant \ell \leqslant m-r .
$$

In other words, $\operatorname{Ann}(\mathcal{G}) \subset \mathbb{F}^{m}$ is flat over $\mathrm{CR}((M))$, that is,

$$
\operatorname{Ann}(\mathcal{G})=\mathbb{F}\left(\operatorname{Ann}(\mathcal{G}) \cap(\operatorname{CR}((M)))^{m}\right)
$$

The next result is the main technical proposition from [LM1], establishing convergence of a formal map which satisfies a certain system of singular, partially formal, equations on a generic manifold.

Proposition 3.2. ([LM1, Proposition 3.1]) Let $M \subset \mathbb{C}^{N}$ be a real-analytic generic submanifold through a point $p \in M$, and $\Theta=\left(\Theta_{1}, \ldots, \Theta_{N^{\prime}}\right)$ be a convergent power series 
mapping with components in $\mathbb{C}\{z-p, \bar{z}-\bar{p}, \lambda, w\}$, where $z \in \mathbb{C}^{N}, w \in \mathbb{C}^{N^{\prime}}$ and $\lambda \in \mathbb{C}^{m}$, with $N^{\prime}, N, m \geqslant 1$. Let $H:\left(\mathbb{C}^{N}, p\right) \rightarrow \mathbb{C}^{N^{\prime}}$ and $G:\left(\mathbb{C}^{N}, p\right) \rightarrow \mathbb{C}^{m}$ be formal holomorphic power series mappings, vanishing at $p$, satisfying

$$
\left.\Theta(z, \bar{z}, \overline{G(z)}, H(z))\right|_{M}=0, \quad \text { and }\left.\quad \operatorname{det} \frac{\partial \Theta}{\partial w}(z, \bar{z}, \overline{G(z)}, H(z))\right|_{M} \not \equiv 0 .
$$

If $M$ is of finite type at $p$, then $H$ is convergent.

Remark 3.3. It is well known that, for every real-analytic holomorphically nondegenerate hypersurface $M \subset \mathbb{C}^{N}$ (in the sense of [Sta]) and every $p \in M$, the formal self-equivalences $H$ of $(M, p)$ satisfy systems of equations of the form (3.1) (see, e.g., [Mir1]). The existence of such hypersurfaces (of infinite type) with divergent formal CR equivalences (see $[\mathrm{KS}]$ ) shows that Proposition 3.2 does not hold in general if the finite type assumption is dropped.

\subsection{The divergence rank}

We equip $\mathbb{C}^{N}$ with coordinates $z=\left(z_{1}, \ldots, z_{N}\right)$, and $\mathbb{C}^{N^{\prime}}$ with coordinates $w=\left(w_{1}, \ldots, w_{N^{\prime}}\right)$. With respect to these coordinates, we decompose our map $H$ into components

$$
H(z)=\left(H_{1}(z), \ldots, H_{N^{\prime}}(z)\right) \in(\mathbb{C}[[z-p]])^{N^{\prime}} .
$$

Let $\mathcal{A}_{H}$ be the set of all pairs $(\Delta, S)$ of power series such that $\Delta=\Delta(z) \in(\mathbb{C}[[z-p]])^{m}$ for some $m$ and

$$
S=S(z, \bar{z}, \lambda, w) \in \mathbb{C}\{z-p, \bar{z}-\bar{p}, \lambda-\overline{\Delta(p)}, w-H(p)\},
$$

where $\lambda \in \mathbb{C}^{m}$. For every $(\Delta, S) \in \mathcal{A}_{H}$, we set

$$
\begin{aligned}
S^{\Delta}: & =\left.S(z, \bar{z}, \overline{\Delta(z)}, H(z))\right|_{M} \in \mathbb{C}[[M]], \\
S_{w_{j}}^{\Delta} & :=\left.\frac{\partial S}{\partial w_{j}}(z, \bar{z}, \overline{\Delta(z)}, H(z))\right|_{M} \in \mathbb{C}[[M]], \quad j=1, \ldots, N^{\prime}, \\
S_{w}^{\Delta} & :=\left(S_{w_{1}}^{\Delta}, \ldots, S_{w_{N^{\prime}}}^{\Delta}\right) \in(\mathbb{C}[[M]])^{N^{\prime}} .
\end{aligned}
$$

We now consider the subring $\mathcal{S}_{H}(M) \subset \mathbb{C}[[M]]$ consisting of those power series of the form $S^{\Delta}$ for some $(\Delta, S) \in \mathcal{A}_{H}$, and let $\mathbb{K}_{H}^{M}$ denote the quotient field of $\mathcal{S}_{H}(M)$.

Finally, we denote by $\mathcal{A}_{H}^{0}(M)$ the subset of $\mathcal{A}_{H}$ consisting of all pairs $(\Delta, S)$ satisfying $S^{\Delta}=0$, and define

$$
\operatorname{rank} \mathcal{A}_{H}^{0}(M):=\operatorname{dim}_{\mathbb{K}_{H}^{M}} \operatorname{span}\left\{S_{w}^{\Delta}:(\Delta, S) \in \mathcal{A}_{H}^{0}(M)\right\},
$$

where the dimension is computed over the field $\mathbb{K}_{H}^{M}$, and where every $S_{w}^{\Delta}$ is considered as a vector in $\left(\mathcal{S}_{H}(M)\right)^{N^{\prime}} \subset\left(\mathbb{K}_{H}^{M}\right)^{N^{\prime}}$. 
Definition 3.4. Let $M$ and $H$ be as above. We define the divergence rank of $H$ by

$$
\operatorname{divrk}_{M} H=N^{\prime}-\operatorname{rank} \mathcal{A}_{H}^{0}(M)
$$

The following result provides a link between the divergence rank of a formal map and its divergence/convergence properties.

Proposition 3.5. Let $M \subset \mathbb{C}^{N}$ be a generic real-analytic submanifold, $p \in M$ and let $H:\left(\mathbb{C}^{N}, p\right) \rightarrow \mathbb{C}^{N^{\prime}}$ be a formal holomorphic map. The following statements hold:

(a) $\operatorname{divrk}_{M} H \leqslant \delta$, where $\delta$ is the number of divergent components of $H$;

(b) if $M$ is of finite type at $p$, then $\operatorname{divrk}_{M} H=0$ if and only if $H$ is convergent.

Proof. In order to prove (a), note that, if $H$ has $\delta$ components that are divergent, say the last $\delta$ ones, and $H_{1}, \ldots, H_{N^{\prime}-\delta}$ are all convergent, then we may set

$$
S^{j}(z, w)=w_{j}-H_{j}(z) \in \mathbb{C}\{z-p, w-H(p)\} \quad \text { for } j=1, \ldots, N^{\prime}-\delta,
$$

and see that each $\left(0, S^{j}\right) \in \mathcal{A}_{H}^{0}(M)$. Hence, $\operatorname{rank} \mathcal{A}_{H}^{0}(M) \geqslant N^{\prime}-\delta$, which proves (a).

In order to prove (b), it remains to check, due to (a), that if $\operatorname{divrk}_{M} H=0$ then $H$ is convergent. For this note that $\operatorname{divrk}_{M} H=0$ means that there exist $\Delta \in(\mathbb{C}[[z-p]])^{m}$ (for some integer $m)$ and $\Theta:=\left(S^{1}, \ldots, S^{N^{\prime}}\right) \in(\mathbb{C}\{z-p, \bar{z}-\bar{p}, \lambda-\overline{\Delta(p)}, w-H(p)\})^{N^{\prime}}$ such that

$$
\left.\Theta(z, \bar{z}, \overline{\Delta(z)}, H(z))\right|_{M}=0 \quad \text { and }\left.\quad \operatorname{det} \Theta_{w}(z, \bar{z}, \overline{\Delta(z)}, H(z))\right|_{M} \not \equiv 0 .
$$

Applying Proposition 3.2, we get that $H$ is convergent.

Remark 3.6. Note that, in Proposition 3.5, the inequality in (a) can be strict: Indeed, if $H=\left(H_{1}, \ldots, H_{N^{\prime}}\right)$, with $H_{1}$ divergent and $H_{2}, \ldots, H_{N^{\prime}}$ convergent, then, after a change of coordinates $\widetilde{Z}_{1}=Z_{1}$ and $\widetilde{Z}_{j}=Z_{j}+Z_{1}, j \geqslant 2$, the map $\widetilde{H}$ has no convergent components, but the divergence rank (being independent of the chosen coordinates) is still at most 1 .

There are also more interesting examples where a strict inequality may occur, such as the following one: Consider the real-algebraic hypersurface $M$ in $\mathbb{C}_{z_{1}, z_{2}, z_{3}}^{3}$ given by $\operatorname{Im} z_{3}=\left|z_{1} z_{2}\right|^{2}$, and the formal holomorphic map $H\left(z_{1}, z_{2}, z_{3}\right)=\left(z_{1} e^{h\left(z_{1}\right)}, z_{2} e^{-h\left(z_{1}\right)}, z_{3}\right)$, where $h\left(z_{1}\right)$ is any formal divergent power series satisfying $h(0)=0$. As $H(M) \subset M$, one can check that $\operatorname{divrk}_{M} H=1$, whereas $H$ has two divergent components.

Remark 3.7. It follows from Remark 3.3 that there exists germs of real-analytic hypersurfaces $(M, p)$ in $\mathbb{C}^{N}$ of infinite type and divergent formal holomorphic maps $H:\left(\mathbb{C}^{N}, p\right) \rightarrow \mathbb{C}^{N}$ such that $\operatorname{divrk}_{M} H=0$. This shows that Proposition 3.5 (b) does not hold in general when the finite type assumption is dropped. 


\subsection{Construction of formal meromorphic vectors}

We define the following vector subspace of $\left(\mathbb{K}_{H}^{M}\right)^{N^{\prime}}$.

$$
\begin{aligned}
\mathcal{V}_{H}^{M}: & =\left\{V=\left(V_{1}, \ldots, V_{N^{\prime}}\right) \in\left(\mathbb{K}_{H}^{M}\right)^{N^{\prime}}: V \cdot S_{w}^{\Delta}=0 \text { for all }(\Delta, S) \in \mathcal{A}_{H}^{0}(M)\right\} \\
& =\operatorname{Ann}\left\{S_{w}^{\Delta}:(\Delta, S) \in \mathcal{A}_{H}^{0}(M)\right\}
\end{aligned}
$$

where

$$
V \cdot S_{w}^{\Delta}=\sum_{j=1}^{N^{\prime}} V_{j} S_{w_{j}}^{\Delta}
$$

The following is the main result of this section.

Proposition 3.8. Let $M \subset \mathbb{C}^{N}$ be a generic real-analytic submanifold, $p \in M$ and $H:\left(\mathbb{C}^{N}, p\right) \rightarrow \mathbb{C}^{N^{\prime}}$ be a formal holomorphic map. For $\mathcal{A}_{H}^{0}(M), \mathcal{V}_{H}^{M}$ and $\operatorname{divrk}_{M} H$ defined as above, we have

$$
\operatorname{dim}_{\mathbb{K}_{H}^{M}} \mathcal{V}_{H}^{M}=\operatorname{divrk}_{M} H,
$$

and there exists a basis of $\mathcal{V}_{H}^{M}$ that consists of $\ell$ CR vectors in $\left(\mathbb{K}_{H}^{M}\right)^{N^{\prime}}$ which are linearly independent over $\mathbb{K}_{H}^{M}$, with $\ell:=\operatorname{divrk}_{M} H$.

Proof. From Definition 3.4, we can find $\left(\Gamma, S_{1}\right), \ldots,\left(\Gamma, S_{N^{\prime}-\ell}\right) \in \mathcal{A}_{H}^{0}(M)$ such that

$$
\operatorname{dim}_{\mathbb{K}_{H}^{M}} \operatorname{span}\left\{S_{j, w}^{\Gamma}: 1 \leqslant j \leqslant N^{\prime}-\ell\right\}=N^{\prime}-\ell
$$

Set

$$
\mathcal{W}_{H}^{M}:=\left\{V=\left(V_{1}, \ldots, V_{N^{\prime}}\right) \in\left(\mathbb{K}_{H}^{M}\right)^{N^{\prime}}: V \cdot S_{j, w}^{\Gamma}=0 \text { for all } 1 \leqslant j \leqslant N^{\prime}-\ell\right\} \supset \mathcal{V}_{H}^{M},
$$

and note that $\operatorname{dim}_{\mathbb{K}_{H}^{M}} \mathcal{W}_{H}^{M}=\ell$.

Let $(\Delta, S) \in \mathcal{A}_{H}^{0}(M)$. By the definition of $\ell$, the vector $S_{w}^{\Delta} \in\left(\mathbb{K}_{H}^{M}\right)^{N^{\prime}}$ is $\mathbb{K}_{H}^{M}$-linearly dependent over the vectors $S_{j, w}^{\Gamma}, 1 \leqslant j \leqslant N^{\prime}-\ell$. This implies that $\mathcal{W}_{H}^{M}=\mathcal{V}_{H}^{M}$, and hence proves (3.4).

Next, we claim that, for any real-analytic CR vector field $\bar{L}$ near $p$, the dimension of the space spanned by all the vectors $S_{j, w}^{\Gamma}$ and $\bar{L} S_{j, w}^{\Gamma}$ for $1 \leqslant j \leqslant N^{\prime}-\ell$ over $\mathbb{K}_{H}^{M}$ is still $N^{\prime}-\ell$. Indeed, we first note that

$$
\bar{L} S_{j, w_{\nu}}^{\Gamma}=\left.\bar{L}\left(\frac{\partial S_{j}}{\partial w_{\nu}}(z, \bar{z}, \overline{\Gamma(z)}, H(z))\right)\right|_{M} .
$$

Since $\left(\Gamma, S_{j}\right) \in \mathcal{A}_{H}^{0}(M)$ for every $1 \leqslant j \leqslant N^{\prime}-\ell$, for each $1 \leqslant \nu \leqslant N^{\prime}$ we may write, using the chain rule,

$$
\bar{L}\left(\frac{\partial S_{j}}{\partial w_{\nu}}(z, \bar{z}, \overline{\Gamma(z)}, w)\right)=\frac{\partial \widetilde{S}_{j}}{\partial w_{\nu}}(z, \bar{z}, \overline{\widetilde{\Gamma}(z)}, w)
$$


for some $\left(\widetilde{\Gamma}, \widetilde{S}_{j}\right) \in \mathcal{A}_{H}^{0}(M)$. It follows that the vectors $\bar{L} S_{j, w}^{\Gamma}$ coincide with $\widetilde{S}_{j, w}^{\widetilde{\Gamma}}$, where $\left(\widetilde{\Gamma}, \widetilde{S}_{j}\right) \in \mathcal{A}_{H}^{0}(M)$ for all $j$. From our choice of $\left(\Gamma, S_{j}\right), 1 \leqslant j \leqslant N^{\prime}-\ell$, the claim follows. Hence, the $\mathbb{K}_{H}^{M}$-vector subspace $\mathcal{G}$ spanned by the $S_{j, w}^{\Gamma}, 1 \leqslant j \leqslant N^{\prime}-\ell$, is closed under the application of $\mathrm{CR}$ vector fields. Applying Lemma 3.1 (with $\mathbb{F}=\mathbb{K}_{H}^{M}$ ), we reach the desired conclusion.

We still need some simple but useful remarks regarding notation for the rest of the paper.

Given $(\Delta, S),(\Delta, T) \in \mathcal{A}_{H}$ such that $(\Delta, T) \notin \mathcal{A}_{H}^{0}(M), S^{\Delta} / T^{\Delta}$ defines an element of $\mathbb{K}_{H}^{M}$. We define in the natural way

$$
\left(\frac{S}{T}\right)_{w}^{\Delta}:=\frac{T^{\Delta} S_{w}^{\Delta}-S^{\Delta} T_{w}^{\Delta}}{\left(T^{\Delta}\right)^{2}} \in\left(\mathbb{K}_{H}^{M}\right)^{N^{\prime}} .
$$

The following is a fact regarding the space $\mathcal{V}_{H}^{M}$ which we will use often; we are indeed going to need it to give meaning to some evaluations of gradients of elements of $\mathbb{K}_{H}^{M}$.

LEMma 3.9. With the above notation, for all $V \in \mathcal{V}_{H}^{H}$ and all $(\Delta, S),(\Delta, T) \in \mathcal{A}_{H}$ such that $(\Delta, S) \in \mathcal{A}_{H}^{0}(M)$ and $(\Delta, T) \notin \mathcal{A}_{H}^{0}(M)$,

$$
V \cdot\left(\frac{S}{T}\right)_{w}^{\Delta}:=\sum_{j=1}^{N^{\prime}} V_{j}\left(\frac{S}{T}\right)_{w_{j}}^{\Delta}=0 .
$$

Now, given $\eta \in \mathbb{K}_{H}^{M}$, there exist $(\Delta, S),(\Delta, T) \in \mathcal{A}_{H}$, with $(\Delta, T) \notin \mathcal{A}_{H}^{0}(M)$, such that $\eta=S^{\Delta} / T^{\Delta}$. One would like to define $\eta_{w}$ as $(S / T)_{w}^{\Delta}$, but such a choice might depend on the chosen representative. In fact, if $(\tilde{\Delta}, \widetilde{S}),(\tilde{\Delta}, \widetilde{T}) \in \mathcal{A}_{H}$ are such that $(\tilde{\Delta}, \widetilde{T}) \notin \mathcal{A}_{H}^{0}(M)$ and $\eta=\widetilde{S}^{\tilde{\Delta}} / \widetilde{T}^{\tilde{\Delta}}$, it is entirely possible that $(S / T)_{w}^{\Delta} \neq(\widetilde{S} / \widetilde{T})_{w}^{\tilde{\Delta}}$. However, Lemma 3.9 shows that, for every $V \in \mathcal{V}_{H}^{M}$, we have

$$
V \cdot\left(\frac{S}{T}\right)_{w}^{\Delta}=V \cdot\left(\frac{\widetilde{S}}{\widetilde{T}}\right)_{w}^{\tilde{\Delta}},
$$

which shows that, even though $\eta_{w}$ is not well defined, $V \cdot \eta_{w}$ is, by the expression on either side of (3.5).

Finally, given a polynomial map

$$
P(t, \bar{t})=\sum_{\alpha, \beta \in \mathbb{N}^{k}} P_{\alpha, \beta} t^{\alpha} \bar{t}^{\beta} \in\left(\mathbb{K}_{H}^{M}[t, \bar{t}]\right)^{c},
$$

$t=\left(t_{1}, \ldots, t_{k}\right), k, c \geqslant 1$, we define, for $V \in \mathcal{V}_{H}^{M}$,

$$
V \cdot P_{w}(t, \bar{t}):=\sum_{\alpha, \beta \in \mathbb{N}^{k}} V \cdot P_{\alpha, \beta ; w} t^{\alpha} \bar{t}^{\beta} \in\left(\mathbb{K}_{H}^{M}[t, \bar{t}]\right)^{c},
$$

where we write $P_{\alpha, \beta ; w}=\left(P_{\alpha, \beta}\right)_{w}$.

We conclude with the following lemma. 
LEMma 3.10. With the above notation, let

$$
P(t, \bar{t})=\sum_{\alpha, \beta \in \mathbb{N}^{k}} P_{\alpha, \beta} t^{\alpha} \bar{t}^{\beta} \in\left(\mathbb{K}_{H}^{M}[t, \bar{t}]\right)^{c}, \quad k, c \geqslant 1
$$

Then, the following statements hold:

(i) if $P(t, \bar{t})=0$, then for every $V \in \mathcal{V}_{H}^{M}$ we have $V \cdot P_{w}(t, \bar{t})=0$;

(ii) if $\bar{L}$ is a $C R$ vector field of $M$ near $p$ with real-analytic coefficients, then

$$
(\bar{L} P)(t, \bar{t}):=\sum_{\alpha, \beta \in \mathbb{N}^{k}}\left(\bar{L} P_{\alpha, \beta}\right) t^{\alpha} \bar{t}^{\beta} \in\left(\mathbb{K}_{H}^{M}[t, \bar{t}]\right)^{c},
$$

and, for any $C R$ vector $V \in \mathcal{V}_{H}^{M}$, we have

$$
V \cdot(\bar{L} P)_{w}(t, \bar{t})=\bar{L}\left(V \cdot P_{w}(t, \bar{t})\right) .
$$

Proof. Part (i) is immediate. Part (ii) is a direct consequence of the following observation. Given $\eta \in \mathbb{K}_{H}^{M}$ and any CR vector $V \in \mathcal{V}_{H}^{M}$, the expression $V \cdot \bar{L}(S / T)_{w}^{\Delta}$ is independent of the choice of representative $\eta=(S / T)^{\Delta}$, and the following identities hold:

$$
\bar{L}\left(V \cdot \eta_{w}\right)=V \cdot \bar{L}\left(\frac{S}{T}\right)_{w}^{\Delta}=V \cdot(\bar{L} \eta)_{w}
$$

\section{From formal meromorphic vectors to approximate formal deformations: proof of Theorem 2.2}

In this section, we will prove Theorem 2.2, that is, we will construct, for any divergent formal CR map, approximate formal deformations of any order. In a first step, we are going to use the formal meromorphic vectors introduced in the previous section to build formal deformations of a special type, i.e. formal deformations with formal meromorphic coefficients (Theorem 4.1 below).

\subsection{Construction of a special type of formal deformations for maps of divergence rank $\geqslant 1$}

TheOREM 4.1. Let $M \subset \mathbb{C}^{N}$ be a generic real-analytic submanifold, let $p \in M$ and assume that $N, N^{\prime} \geqslant 2$. Let $H:\left(\mathbb{C}^{N}, p\right) \rightarrow \mathbb{C}^{N^{\prime}}$ be a formal holomorphic map with $r=$ $\operatorname{divrk}_{M} H \in\left\{1, \ldots, N^{\prime}\right\}$, and let $\mathbb{V}=\left(V^{1}, \ldots, V^{r}\right)$ be a basis, containing only $C R$ vectors, of $\mathcal{V}_{H}^{M}$ over $\mathbb{K}_{H}^{M}$ as given in Proposition 3.8. For $t=\left(t_{1}, \ldots, t_{r}\right) \in \mathbb{C}^{r}$ set $t \cdot \mathbb{V}=\sum_{i=1}^{r} t_{i} V^{i}$. 
We inductively define, for every $\ell \in \mathbb{Z}_{+}$, a homogeneous polynomial map of degree $\ell$ in $\left(\mathbb{K}_{H}^{M}[t]\right)^{N^{\prime}}$ as follows:

$$
D^{1}(t):=t \cdot \mathbb{V}, \quad D^{\ell+1}(t)=\frac{1}{\ell+1}(t \cdot \mathbb{V}) \cdot D_{w}^{\ell}(t),
$$

and set

$$
D(t)=\sum_{\ell=1}^{\infty} D^{\ell}(t) \in\left(\mathbb{K}_{H}^{M}[[t]]\right)^{N^{\prime}} .
$$

Then, the following statements hold:

(i) $D(t) \in(\mathrm{CR}((M))[[t]])^{N^{\prime}}$;

(ii) if $\varrho \in \mathbb{C}\{w-H(p), \bar{w}-\overline{H(p)}\}$ satisfies $\left.\varrho(H(z), \overline{H(z)})\right|_{M}=0$, then

$$
\varrho(H+D(t), \overline{H+D(t)})=0 \quad \text { in } \mathbb{C}((M))[[t, \bar{t}]] .
$$

Proof. The fact that the polynomial maps $D^{\ell}(t)$ are homogeneous of degree $\ell$ with coefficients in $\mathbb{K}_{H}^{M}$ is clear from the given construction. The proof of Theorem 4.1 is based on three main ingredients: the properties of the space $\mathcal{V}_{H}^{M}$ previously described in $\S 3.3$, the chain rule, and the crucial property stating that, for every $i=1, \ldots, r$, the components of $\overline{V^{i}}$ still belong to $\mathbb{K}_{H}^{M}$, a property following from the fact that the $V^{i}$, are $\mathrm{CR}$ vectors.

To prove (i), we need to check that $D^{\ell}(t) \in(\mathrm{CR}((M))[t])^{N^{\prime}}$ for every $\ell \geqslant 1$, which we will prove by induction on $\ell$. For $\ell=1$, this follows from the fact that $\mathbb{V}$ is a set of $r \mathrm{CR}$ vectors in $\mathbb{C}((M))$. Assume now that $D^{\ell}(t) \in(\mathrm{CR}((M))[t])^{N^{\prime}}$ for some $\ell$, that is, for every real-analytic CR vector field $\bar{L}$ near $p$, we have $\left(\bar{L} D^{\ell}\right)(t)=0$ in $\left(\mathbb{K}_{H}^{M}[t]\right)^{N^{\prime}}$. From the definition of $\mathcal{V}_{H}^{M}$ and Lemma 3.9, we have $V^{i} \cdot\left(\bar{L} D^{\ell}\right)_{w}(t)=0$ for $1 \leqslant i \leqslant r$. Using Lemma 3.10 (ii), we obtain

$$
0=V^{i} \cdot\left(\bar{L} D^{\ell}\right)_{w}(t)=\bar{L}\left(V^{i} \cdot D_{w}^{\ell}(t)\right), \quad 1 \leqslant i \leqslant r .
$$

This proves $V^{i} \cdot D_{w}^{\ell}(t) \in(\mathrm{CR}((M))[t])^{N^{\prime}}$ for every $i$, and therefore $D^{\ell+1}(t) \in(\mathrm{CR}((M))[t])^{N^{\prime}}$, too. This completes the proof of (i).

It remains to prove (ii). To this aim, we need the following lemma.

Lemma 4.2. Let $\varrho, H$ and $D$ be as in Theorem 4.1. Write

$$
\varrho(H+D(t), \overline{H+D(t)})=\sum_{j, k \in \mathbb{Z}_{+}} \frac{R^{j, k}(t, \bar{t})}{j ! k !} \in \mathbb{C}((M))[[t, \bar{t}]],
$$

where each $R^{j, k}$ is homogeneous of degree $j$ in $t$, and of degree $k$ in $\bar{t}$. Then, for any $j, k \in \mathbb{Z}_{+}$, there exists a universal polynomial $\mathcal{U}_{j, k}$ in all its arguments such that

$$
R^{j, k}(t, \bar{t})=\mathcal{U}_{j, k}\left(\left(\varrho_{w^{\beta}} \bar{w}^{\nu}(H, \bar{H})\right)_{\substack{|\beta| \leqslant j \\|\nu| \leqslant k}},\left(\ell ! D^{\ell}(t)\right)_{\ell \leqslant j},\left(s ! \overline{D^{s}(t)}\right)_{s \leqslant k}\right) .
$$


Furthermore, for $j, k \in \mathbb{Z}_{+}$, writing

$$
\mathcal{U}_{j, k}=\mathcal{U}_{j, k}\left(\left(\Lambda_{\beta, \nu}\right)_{\substack{|\beta| \leqslant j \\|\nu| \leqslant k}}, X_{1}, \ldots, X_{j}, Y_{1}, \ldots, Y_{k}\right)
$$

where $\Lambda_{\beta, \nu} \in \mathbb{C}$ and $X_{i}, Y_{m} \in \mathbb{C}^{N^{\prime}}$, we have

$$
\begin{aligned}
& R^{j+1, k}(t, \bar{t}) \\
& =\sum_{i=1}^{j}(i+1) ! \frac{\partial \mathcal{U}_{j, k}}{\partial X_{i}}\left(\left(\varrho_{w^{\beta} \bar{w}^{\nu}}(H, \bar{H})\right)_{\substack{|\beta| \leqslant j \\
|\nu| \leqslant k}},\left(\ell ! D^{\ell}(t)\right)_{\ell \leqslant j},\left(s ! \overline{D^{s}(t)}\right)_{s \leqslant k}\right) \cdot D^{i+1}(t) \\
& \quad+\sum_{\substack{|\gamma| \leqslant j \\
|\mu| \leqslant k}} \frac{\partial \mathcal{U}_{j, k}}{\partial \Lambda_{\gamma, \mu}}\left(\left(\varrho_{w^{\beta} \bar{w}^{\nu}}(H, \bar{H})\right)_{\substack{|\beta| \leqslant j \\
|\nu| \leqslant k}},\left(\ell ! D^{\ell}(t)\right)_{\ell \leqslant j},\left(s ! \overline{D^{s}(t)}\right)_{s \leqslant k}\right) \\
& \quad \times D^{1}(t) \cdot\left(\varrho_{w^{\gamma} \bar{w}^{\mu}}(H, \bar{H})\right)_{w}
\end{aligned}
$$

Proof. The proof is a consequence of the chain rule. The details are as follows.

In what follows, we write $D(t)=\left(D_{1}(t), \ldots, D_{N^{\prime}}(t)\right)$ and $\mathcal{D}(t)=H+D(t)$. Consider, for $\lambda \in \mathbb{C}$,

$$
\Phi(t, \bar{t}, \lambda, \bar{\lambda})=\varrho(\mathcal{D}(\lambda t), \overline{\mathcal{D}(\lambda t)})=\sum_{j, k \in \mathbb{Z}_{+}} \frac{R^{j, k}(t, \bar{t})}{j ! k !} \lambda^{j} \bar{\lambda}^{k} \in \mathbb{C}((M))[[t, \bar{t}, \lambda, \bar{\lambda}]],
$$

and hence, for every $j, k \in \mathbb{Z}_{+}$,

$$
R^{j, k}(t, \bar{t})=\Phi_{\lambda^{j} \bar{\lambda}^{k}}(t, \bar{t}, 0,0) .
$$

Fix $j$ and $k$. By the chain rule, there exists a universal polynomial of its arguments $\mathcal{U}_{j, k}$ such that

$$
\begin{aligned}
& \Phi_{\lambda^{j} \bar{\lambda}^{k}}(t, \bar{t}, \lambda, \bar{\lambda}) \\
& \quad=\mathcal{U}_{j, k}\left(\left(\varrho_{w^{\beta} \bar{w}^{\nu}}(\mathcal{D}(\lambda t), \overline{\mathcal{D}(\lambda t)})\right)_{\substack{|\beta| \leqslant j \\
|\nu| \leqslant k}},\left(\partial_{\lambda}^{\ell}(D(\lambda t))\right)_{1 \leqslant \ell \leqslant j},\left(\partial_{\bar{\lambda}}^{s}(\overline{D(\lambda t)})\right)_{1 \leqslant s \leqslant k}\right) .
\end{aligned}
$$

Setting $\lambda=0$ in (4.6) and using (4.5) yields (4.3). Now, differentiating (4.6) with respect to $\lambda$ yields that

$$
\begin{gathered}
\Phi_{\lambda^{j+1} \bar{\lambda}^{k}}(t, \bar{t}, \lambda, \bar{\lambda}) \\
=\sum_{i=1}^{j} \frac{\mathcal{U}_{j, k}}{\partial X_{i}}\left(\left(\varrho_{w^{\beta} \bar{w}^{\nu}}\right)_{\substack{|\beta| \leqslant j \\
|\nu| \leqslant k}},\left(\partial_{\lambda}^{\ell}(D(\lambda t))\right)_{1 \leqslant \ell \leqslant j},\left(\partial_{\bar{\lambda}}^{s}(\overline{D(\lambda t)})\right)_{1 \leqslant s \leqslant k}\right) \cdot \partial_{\lambda}^{i+1}(D(\lambda t)) \\
+\sum_{\substack{|\gamma| \leqslant j \\
|\mu| \leqslant k}} \frac{\partial \mathcal{U}_{j, k}}{\partial \Lambda_{\gamma, \mu}}\left(\left(\varrho_{w^{\beta}} \bar{w}^{\nu}\right)_{\substack{|\beta| \leqslant j \\
|\nu| \leqslant k}},\left(\partial_{\lambda}^{\ell}(D(\lambda t))\right)_{\ell \leqslant j},\left(\partial_{\bar{\lambda}}^{s}(\overline{D(\lambda t)})\right)_{s \leqslant k}\right) \\
\times \sum_{m=1}^{N^{\prime}} \varrho_{w^{\gamma} w_{m} \bar{w}^{\mu}} \partial_{\lambda}\left(D_{m}(\lambda t)\right),
\end{gathered}
$$




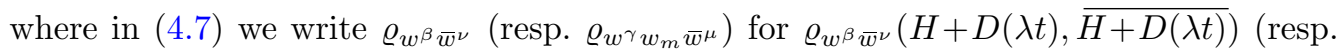
$\left.\varrho_{w^{\gamma} w_{m} \bar{w}^{\mu}}(H+D(\lambda t), \overline{H+D(\lambda t)})\right)$. Setting $\lambda=0$ in (4.7) and using that

$$
\left.\partial_{\lambda}^{\ell}(D(\lambda t))\right|_{\lambda=0}=\ell ! D^{\ell}(t)
$$

for every integer $\ell$ yields (4.4). This completes the proof of the lemma.

We can now continue the proof of Theorem 4.1 (ii). We will show that $R^{j, k}(t, \bar{t})=0$, by induction on $e=k+j$, where we recall that $R^{j, k}(t, \bar{t})$ is given in (4.2). First, note that $R^{0,0}(t, \bar{t})=\left.\varrho(H, \bar{H})\right|_{M}=0$. Hence, let $e \in \mathbb{Z}_{+}$and let us assume that $R^{j, k}(t, \bar{t})=0$ for all $j+k \leqslant e$ and prove that $R^{j+1, k}(t, \bar{t})=R^{j, k+1}(t, \bar{t})=0$ for all $j$ and $k$ such that $j+k \leqslant e$.

By Lemma 4.2 , there exists a universal polynomial $\mathcal{U}_{j, k}$ in all its arguments such that

$$
0=R^{j, k}(t, \bar{t})=\mathcal{U}_{j, k}\left(\left(\varrho_{w^{\beta} \bar{w}^{\nu}}(H, \bar{H})\right)_{\substack{|\beta| \leqslant j \\|\nu| \leqslant k}},\left(\ell ! D^{\ell}(t)\right)_{\ell \leqslant j},\left(s ! \overline{D^{s}(t)}\right)_{s \leqslant k}\right) .
$$

Note that $\overline{\mathrm{CR}((M))} \subset \mathbb{K}_{H}^{M}$. Because each $D^{\ell}(t)$ lies at the same time in $\left(\mathbb{K}_{H}^{M}[t]\right)^{N^{\prime}}$ and in $(\mathrm{CR}((M))[t])^{N^{\prime}}$, we have $R^{j, k}(t, \bar{t}) \in \mathbb{K}_{H}^{M}[t, \bar{t}]$. Hence, (4.8) together with Lemma 3.10 (i) implies that

$$
D^{1}(t) \cdot R_{w}^{j, k}(t, \bar{t})=(t \cdot \mathbb{V}) \cdot R_{w}^{j, k}(t, \bar{t})=0 .
$$

In view of (4.8) and using (4.1), we have

$$
\begin{aligned}
& D^{1}(t) \cdot R_{w}^{j, k}(t, \bar{t}) \\
& =\sum_{i=1}^{j} i ! \frac{\partial \mathcal{U}_{j, k}}{\partial X_{i}}\left(\left(\varrho_{w^{\beta} \bar{w}^{\nu}}(H, \bar{H})\right)_{\substack{|\beta| \leqslant j \\
|\nu| \leqslant k}},\left(\ell ! D^{\ell}(t)\right)_{\ell \leqslant j},\left(s ! \overline{D^{s}(t)}\right)_{s \leqslant k}\right) \cdot D^{1}(t) \cdot D_{w}^{i}(t) \\
& \quad+\sum_{\substack{|\gamma| \leqslant j \\
|\mu| \leqslant k}} \frac{\partial \mathcal{U}_{j, k}}{\partial \Lambda_{\gamma, \mu}}\left(\left(\varrho_{w^{\beta} \bar{w}^{\nu}}(H, \bar{H})\right)_{\substack{|\beta| \leqslant j \\
|\nu| \leqslant k}},\left(\ell ! D^{\ell}(t)\right)_{\ell \leqslant j},\left(s ! \overline{D^{s}(t)}\right)_{s \leqslant k}\right) \\
& \quad \times D^{1}(t) \cdot\left(\varrho_{w^{\gamma} \bar{w}^{\mu}}(H, \bar{H})\right)_{w} \\
& =\sum_{i=1}^{j}(i+1) ! \frac{\partial \mathcal{U}_{j, k}}{\partial X_{i}}\left(\left(\varrho_{w^{\beta} \bar{w}^{\nu}}(H, \bar{H})\right)_{\substack{|\beta| \leqslant j \\
|\nu| \leqslant k}},\left(\ell ! D^{\ell}(t)\right)_{\ell \leqslant j},\left(s ! \overline{D^{s}(t)}\right)_{s \leqslant k}\right) \cdot D^{i+1}(t) \\
& \quad+\sum_{\substack{|\gamma| \leqslant j \\
|\mu| \leqslant k}} \frac{\partial \mathcal{U}_{j, k}}{\partial \Lambda_{\gamma, \mu}}\left(\left(\varrho_{w^{\beta} \bar{w}^{\nu}}(H, \bar{H})\right)_{\substack{|\beta| \leqslant j \\
|\nu| \leqslant k}},\left(\ell ! D^{\ell}(t)\right)_{\ell \leqslant j},\left(s ! \overline{D^{s}(t)}\right)_{s \leqslant k}\right) \\
& \times D^{1}(t) \cdot\left(\varrho_{w^{\gamma} \bar{w}^{\mu}}(H, \bar{H})\right)_{w} .
\end{aligned}
$$

Hence, using (4.4) in Lemma 4.2, we obtain that

$$
D^{1}(t) \cdot R_{w}^{j, k}(t, \bar{t})=R^{j+1, k}(t, \bar{t}) .
$$


Using (4.9), we get that $R^{j+1, k}(t, \bar{t})=0$ for $j+k \leqslant e$. In order to prove that $R^{j, k+1}(t, \bar{t})=0$ for $j+k \leqslant e$, it suffices to observe the following. Consider the power series

$$
\varphi \in \mathbb{C}\{w-H(p), \bar{w}-\overline{H(p)}\}
$$

given by $\varphi(w, \bar{w})=\overline{\varrho(w, \bar{w})}$. Then, $\left.\varphi(H, \bar{H})\right|_{M}=0$, and one has

$$
\varphi(H+D(t), \overline{H+D(t)})=\sum_{m, n \in \mathbb{Z}_{+}} \frac{S^{m, n}(t, \bar{t})}{m ! n !} \in \mathbb{C}((M))[[t, \bar{t}]],
$$

where $S^{m, n}(t, \bar{t})$ is homogeneous of degree $m$ in $t$ and of degree $n$ in $\bar{t}$, and where $S^{m, n}(t, \bar{t})=\overline{R^{n, m}(t, \bar{t})}$. Since $R^{j, k}(t, \bar{t})=0$ for $j+k \leqslant e$, we also have $S^{k, j}(t, \bar{t})=0$ for all such $k$ and $j$. The first part of the proof, applied to $\varphi$ and the $S^{k, j}$, shows that $S^{k+1, j}(t, \bar{t})=0$, and it follows that $R^{j, k+1}(t, \bar{t})=$ for all $j$ and $k$ such that $j+k \leqslant e$. This completes the induction step in the proof of (ii), and therefore the proof of Theorem 4.1.

\subsection{Proof of Theorem 2.2}

Since $M$ is of finite type and $H$ is divergent, it follows from Proposition 3.5 (b) that $r:=\operatorname{divrk}_{M} H \in\left\{1, \ldots, N^{\prime}\right\}$. Let $D(t)$ be given by Theorem 4.1. Let $M^{\prime} \subset \mathbb{C}^{N^{\prime}}$ be a realanalytic set through $H(p)$ such that $H(M) \subset M^{\prime}$. Let also $\varrho \in \mathbb{C}\{w-H(p), \bar{w}-\overline{H(p)}\}$ be any real-analytic function defined near $H(p)$, vanishing on $M^{\prime}$. As $\left.\varrho(H(z), \overline{H(z)})\right|_{M}=0$, we get from Theorem 4.1 that

$$
\varrho(H+D(t), \overline{H+D(t)})=0 \quad \text { in } \mathbb{C}((M))[[t, \bar{t}]] .
$$

Fix $k \in \mathbb{Z}_{+}$. As each coefficient of each component of $D(t)$ is in $\operatorname{CR}((M))$, there exists a non-zero power series $g_{k}(z) \in \mathbb{C}[[z]]$ and power series maps $N_{\gamma}=N_{\gamma}(z) \in(\mathbb{C}[[z]])^{N^{\prime}}, \gamma \in \mathbb{N}^{N}$, $1 \leqslant|\gamma| \leqslant k$, such that

$$
\sum_{\ell=1}^{k} D^{\ell}(t)=\left.\sum_{1 \leqslant|\gamma| \leqslant k} \frac{N_{\gamma}(z)}{g_{k}(z)}\right|_{M} t^{\gamma}
$$

Hence, setting

$$
B^{k}(z, t):=H(z)+\sum_{\ell=1}^{k} D^{\ell}\left(g_{k}(z) t\right)=H(z)+\sum_{1 \leqslant|\gamma| \leqslant k} N_{\gamma}(z)\left(g_{k}(z)\right)^{|\gamma|-1} t^{\gamma}
$$

we have that $B^{k}(z, t) \in(\mathbb{C}[[z, t]])^{N^{\prime}}$ and $B^{k}(z, 0)=H(z)$, and it follows from (4.11) that

$$
\left.\varrho\left(B^{k}(z, t), \overline{B^{k}(z, t)}\right)\right|_{z \in M}=O\left(|t|^{k+1}\right) .
$$


Observe that $B^{k}$ depends only on $H$ and $M$ (and not on $M^{\prime}$ ). Finally, note that, since $D^{1}(t)=t \cdot \mathbb{V}$, where $\mathbb{V}=\left(V^{1}, \ldots, V^{r}\right)$ is of rank $r$ (over $\mathbb{C}((M))$ ), it follows from the above construction that the rank of

$$
\frac{\partial B^{k}}{\partial t}(z, 0)
$$

over $\mathbb{C}((z))$ is also equal to $r$. Hence, $B^{k}$ is a $k$-approximate formal holomorphic deformation for $\left(M, M^{\prime}\right)$ satisfying $B^{k}(z, 0)=H(z)$. This completes the proof of the theorem.

\section{Proofs of Corollaries 2.3 and 2.4}

In order to prove Corollary 2.3 (and hence Corollaries 2.4 and 2.5 and Theorem 1.1), we need to relate the conclusion given in Theorem 2.2 to some geometric properties of the target real-analytic set $M^{\prime}$. Roughly speaking, Theorem 2.2 provides, for any formal divergent map $H:(M, p) \rightarrow M^{\prime}$, a real-analytic CR family (over $M$ ) of formal holomorphic subvarieties tangent to $M^{\prime}$ along the formal "image" of $H$ (along $M$ ), up to any prescribed order. From such a family, we essentially wish to conclude, if possible, the existence of a real-analytic CR family (over $M$ ) of holomorphic complex-analytic subvarieties entirely contained in $M^{\prime}$ and closely related to the original formal map $H$. One possible conclusion one could expect was already mentioned in Conjecture 2.12, but seems out to reach to the authors for the moment. Instead, we will be able to conclude from Theorem 2.2 the statement given in Corollary 2.3 regarding the existence of suitable families of complex-analytic subvarieties in $M^{\prime}$, but with no information on the dependence of the subvarieties on the parameter. Our main tool will be a parameter version of an approximation theorem due to Hickel-Rond [HR].

\subsection{Strong approximation and Hickel-Rond's theorem with parameters}

We start by recalling the following theorem of Hickel-Rond [HR] providing a stronger version of an older result due to Milman [Mil].

Theorem 5.1. ([HR]) Let $R_{1}, \ldots, R_{m} \in \mathbb{C}\{t, \bar{t}, \zeta, \bar{\zeta}\}$, where $t \in \mathbb{C}^{n_{2}}$ and $\zeta \in \mathbb{C}^{n_{3}}$. Then, there exists a function $\mathcal{L}: \mathbb{N} \rightarrow \mathbb{N}$ such that the following holds: If $S(t) \in(\mathbb{C}\{t\})^{n_{3}}$ satisfies $S(0)=0$ and

$$
R_{j}(t, \bar{t}, S(t), \overline{S(t)})=O\left(|t|^{\mathcal{L}(k)+1}\right), \quad j=1, \ldots, m,
$$

for some $k \in \mathbb{N}$, then there exists $\widetilde{S}(t) \in(\mathbb{C}\{t\})^{n_{3}}$ such that

$$
R_{j}(t, \bar{t}, \widetilde{S}(t), \overline{\widetilde{S}(t)})=0, \quad j=1, \ldots, m,
$$

and $S(t)-\widetilde{S}(t)=O\left(|t|^{k+1}\right)$. 
Such kind of statements are usually known in the literature around Artin's approximation theorem as of "strong approximation" type (see [W], the extensive survey by Rond on this matter [Ron], and the survey by Hauser $[\mathrm{H}]$ ). Very closely related problems in the CR setting are also fully discussed in the second author's survey [Mir3].

We will need the following parameter version of Theorem 5.1, which follows from an inspection of its proof.

Theorem 5.2. Let $R_{1}, \ldots, R_{m} \in \mathbb{C}\{u-q, \bar{u}-\bar{q}, t, \bar{t}, \zeta, \bar{\zeta}\}$, where $u \in \mathbb{C}^{n_{1}}, t \in \mathbb{C}^{n_{2}}, \zeta \in$ $\mathbb{C}^{n_{3}}$ and $q \in \mathbb{C}^{n_{1}}$ is fixed. Then, there exist an open neighbourhood $V$ of $q$ in $\mathbb{C}^{n_{1}}$ and a function $\mathcal{L}: \mathbb{N} \rightarrow \mathbb{N}$ such that the following holds: For every $u \in V$, if $S(t) \in(\mathbb{C}\{t\})^{n_{3}}$ satisfies $S(0)=0$ and

$$
R_{j}(u, \bar{u}, t, \bar{t}, S(t), \overline{S(t)})=O\left(|t|^{\mathcal{L}(k)+1}\right), \quad j=1, \ldots, m,
$$

for some $k \in \mathbb{N}$, then there exists $\widetilde{S}(t) \in(\mathbb{C}\{t\})^{n_{3}}$ such that

$$
R_{j}(u, \bar{u}, t, \bar{t}, \widetilde{S}(t), \overline{\widetilde{S}}(t))=0, \quad j=1, \ldots, m
$$

and $S(t)-\widetilde{S}(t)=O\left(|t|^{k+1}\right)$.

Proof. The arguments provided here are due to Guillaume Rond. By [HR], there exist a neighbourhood $\omega$ of $q$ in $\mathbb{C}^{n_{1}}$ and, for $u \in \omega$, a function $\mathcal{L}_{u}: \mathbb{N} \rightarrow \mathbb{N}$ such that the statement of Theorem 5.2 holds, with $\mathcal{L}$ replaced by $\mathcal{L}_{u}$. In order to prove the theorem, one only needs to check that there exists a neighbourhood $V \subset \omega$ of $q$ in $\mathbb{C}^{n_{1}}$ such that the map $V \ni u \mapsto \mathcal{L}_{u}(k)$ is bounded for every integer $k$. This can be proved by repeating the proof given in $[\mathrm{HR}]$, based on the theory of ultraproducts developed by Denef-Lipschitz [DL]. The boundedness of $\mathcal{L}_{u}(k)$, for $u \in V$ and $k \in \mathbb{Z}_{+}$, can be obtained by following step by step the arguments of [DL, p. 26 and Theorem 8.2], as long as the neighbourhood $V \subset \mathbb{C}^{n_{1}} \simeq \mathbb{R}^{2 n_{1}}$ is chosen in such a way that the ring of functions that are real-analytic in a neighbourhood of $\bar{V}$ is Noetherian. According to [Fri, Theorem 1.9], such a choice is possible by taking $V$ to be, e.g., a polydisc. The proof is complete.

\subsection{Proof of Corollary 2.3}

Let $\left(M, M^{\prime}, H\right)$ be as in Corollary 2.3. We may choose a real-analytic function

$$
\varrho=\varrho(w, \bar{w}) \in \mathbb{R}\{w-H(p), \bar{w}-\overline{H(p)}\}
$$

so that the zero-set of $\varrho$ coincides with $M^{\prime}$ near $H(p)$. For

$$
R(u, \bar{u}, \zeta, \bar{\zeta}):=\varrho(u+\zeta, \bar{u}+\bar{\zeta}) \in \mathbb{C}\{u-H(p), \bar{u}-\overline{H(p)}, \zeta, \bar{\zeta}\}, \quad u, \zeta \in \mathbb{C}^{N^{\prime}}
$$


let $V$ be a neighbourhood of $H(p)$ in $\mathbb{C}^{N^{\prime}}$, and $\mathcal{L}$ be given by applying Theorem 5.2 to $R$. We may assume, without loss of generality, that $\mathcal{L}(1) \geqslant 1$. By Theorem 2.2 , there exists $r \in\left\{1, \ldots, N^{\prime}\right\}$ and a formal holomorphic map $B:\left(\mathbb{C}^{N} \times \mathbb{C}^{r},(p, 0)\right) \rightarrow \mathbb{C}^{N^{\prime}}$ such that $B$ is an $\mathcal{L}(1)$-approximate formal deformation of $H$, i.e. such that

$$
\left.\varrho(B(z, t), \overline{B(z, t)})\right|_{z \in M}=O\left(|t|^{\mathcal{L}(1)+1}\right) .
$$

We may write, without loss of generality,

$$
B(z, t)=B_{0}(z)+\sum_{\substack{\gamma \in \mathbb{N}^{N} \\ 1 \leqslant|\gamma| \leqslant \mathcal{L}(1)}} B_{\gamma}(z) t^{\gamma}, \quad B_{0}(z)=H(z) .
$$

Furthermore, since $(\partial B / \partial t)(z, 0)$ is of rank $r$, the same is true for $\left.(\partial B / \partial t)(z, 0)\right|_{M}$. Hence, the matrix of formal power series $\left.(\partial B / \partial t)(z, 0)\right|_{M}$ possesses an $r \times r$ minor that is not zero; we denote the order of vanishing of this minor by $m_{0} \in \mathbb{N}$.

Observe now that (5.1) is equivalent to

$$
\left.\Psi\left(\left(B_{\gamma}(z)\right)_{1 \leqslant|\gamma| \leqslant \mathcal{L}(1)},\left(\overline{B_{\gamma}(z)}\right)_{1 \leqslant|\gamma| \leqslant \mathcal{L}(1)}, B_{0}(z), \overline{B_{0}(z)}\right)\right|_{z \in M}=0
$$

where $\Psi$ is a certain $\mathbb{C}^{\delta}$-valued mapping (for some integer $\delta$ ) whose components are in the ring

$$
\mathbb{C}\{w-H(p), \bar{w}-\overline{H(p)}\}\left[\left(\Lambda_{\gamma}\right)_{1 \leqslant|\gamma| \leqslant \mathcal{L}(1)},\left(\bar{\Lambda}_{\gamma}\right)_{1 \leqslant|\gamma| \leqslant \mathcal{L}(1)}\right],
$$

with each $\Lambda_{\gamma} \in \mathbb{C}^{N^{\prime}}$. By Artin's approximation theorem [A], there exists, for every positive integer $k$ and every $\gamma \in \mathbb{N}^{N}$ with $|\gamma| \leqslant \mathcal{L}(1)$, a convergent power series map

$$
\widehat{B}_{\gamma}^{k}(z, \bar{z}) \in(\mathbb{C}\{z-p, \bar{z}-\bar{p}\})^{N^{\prime}}
$$

such that $\left.\widehat{B}_{\gamma}^{k}(z, \bar{z})\right|_{M}$ agrees with $\left.B_{\gamma}(z)\right|_{M}$ up to order $k$ at $p$, and such that

$$
\left.\Psi\left(\left(\widehat{B}_{\gamma}^{k}(z, \bar{z})\right)_{1 \leqslant|\gamma| \leqslant \mathcal{L}(1)},\left(\overline{\widehat{B}_{\gamma}^{k}(z, \bar{z})}\right)_{1 \leqslant|\gamma| \leqslant \mathcal{L}(1)}, \widehat{B}_{0}^{k}(z, \bar{z}), \overline{\widehat{B}_{0}^{k}(z, \bar{z})}\right)\right|_{M}=0
$$

Hence, setting

$$
S^{k}(z, \bar{z}, t):=\sum_{\substack{\gamma \in \mathbb{N}^{N} \\ 1 \leqslant|\gamma| \leqslant \mathcal{L}(1)}} \widehat{B}_{\gamma}^{k}(z, \bar{z}) t^{\gamma},
$$

we get, for every positive integer $k$,

$$
\begin{aligned}
& \left.R\left(\widehat{B}_{0}^{k}(z, \bar{z}), \overline{\widehat{B}_{0}^{k}(z, \bar{z})}, S^{k}(z, \bar{z}, t), \overline{S^{k}(z, \bar{z}, t)}\right)\right|_{z \in M} \\
& \quad:=\left.\varrho\left(\widehat{B}_{0}^{k}(z, \bar{z})+S^{k}(z, \bar{z}, t), \overline{\widehat{B}_{0}^{k}(z, \bar{z})+S^{k}(z, \bar{z}, t)}\right)\right|_{z \in M}=O\left(|t|^{\mathcal{L}(1)+1}\right) .
\end{aligned}
$$


As $\widehat{B}_{0}^{k}(p, \bar{p})=H(p)$, we may choose a neighbourhood $U_{k}$ of $p$ in $\mathbb{C}^{N}$ such that $M \cap U_{k}$ is connected and $\widehat{B}_{0}^{k}\left(M \cap U_{k}\right) \subset V$. Hence, from the choice of $V$ and $\mathcal{L}$, Theorem 5.2 and (5.4), we get that, for every $z \in M \cap U_{k}$, there exists $\widetilde{S}_{z}^{k}(t) \in(\mathbb{C}\{t\})^{N^{\prime}}$ such that

$$
\left.\varrho\left(\widehat{B}_{0}^{k}(z, \bar{z})+\widetilde{S}_{z}^{k}(t), \overline{\widehat{B}_{0}^{k}(z, \bar{z})+\widetilde{S}_{z}^{k}(t)}\right)\right|_{z \in M \cap U_{k}}=0
$$

satisfying in addition $\widetilde{S}_{z}^{k}(0)=0$ and

$$
\frac{\partial \widetilde{S}_{z}^{k}}{\partial t}(0)=\frac{\partial S^{k}}{\partial t}(z, \bar{z}, 0) .
$$

Now, observe that, if we set $h_{k}:=\widehat{B}_{0}^{k}$, then $h_{k}$ is a real-analytic map that sends, according to (5.5) (for $t=0), M \cap U_{k}$ into $M^{\prime}$. Furthermore, $\left.h_{k}\right|_{M \cap U_{k}}$ agrees with $\left.H\right|_{M}$ at $p$ up to order $k$. Hence, one can find, shrinking $U_{k}$ if necessary, a real-analytic map $U_{k} \rightarrow \mathbb{C}^{N^{\prime}}$ that agrees with $H$ up to order $k$ at $p$ and that coincides with $h_{k}$ on $M \cap U_{k}$. Calling still this new map $h_{k}$, we see that the proof of part (a) of Corollary 2.3 is now complete.

Regarding part (b), recall that

$$
\frac{\partial \widetilde{S}_{z}^{k}}{\partial t}(0)=\frac{\partial S^{k}}{\partial t}(z, \bar{z}, 0) \quad \text { for } z \in M \cap U_{k}
$$

and that

$$
\left.\frac{\partial S^{k}}{\partial t}(z, \bar{z}, 0)\right|_{M} \text { and }\left.\frac{\partial B}{\partial t}(z, 0)\right|_{z \in M}
$$

agree up to order $k$ at $p$. Hence from our above choice of $m_{0}$, it follows that, for $k \geqslant m_{0}+1$, $\left.\left(\partial S^{k} / \partial t\right)(z, \bar{z}, 0)\right|_{M}$ is of rank $r$. This implies that there is a Zariski open subset $\Omega_{k}$ of $M \cap U_{k}$ such the rank of $\left(\partial \widetilde{S}_{z}^{k} / \partial t\right)(0)$ is equal to $r$ for every $z \in \Omega_{k}$. For all such $z$ 's, $(5.5)$ shows that the $r$-dimensional complex submanifold parameterized by $t \mapsto h_{k}(z, \bar{z})+\widetilde{S}_{z}^{k}(t)$ passing through $h_{k}(z, \bar{z})$ is entirely contained in $M^{\prime}$ and, therefore, that $h_{k}(z, \bar{z}) \in \widetilde{\mathcal{E}}_{M^{\prime}}^{r}$, which completes the proof of (b).

Finally, to conclude that $h_{k}\left(M \cap U_{k}\right) \subset \mathcal{E}_{M^{\prime}}$, it suffices to note that $\tilde{\mathcal{E}}_{M^{\prime}}^{r} \subset \mathcal{E}_{M^{\prime}}$, and then invoke the closedness of the set $\mathcal{E}_{M^{\prime}}$ (see [D2] and [D3]).

\subsection{Proof of Corollary 2.4}

This is a direct and simple consequence of Corollary 2.3. Indeed, let $\left(h_{k}\right)_{k \in \mathbb{N}}$ be given by that corollary. From (a), we conclude that there exists an integer $k_{0}$ such that the generic rank of $\left.h_{k_{0}}\right|_{M \cap U_{k_{0}}}$ is at least $\left.\operatorname{Rk} H\right|_{M}$ (where we assumed, without loss of generality, that $M \cap U_{k_{0}}$ is connected). The conclusion of Corollary 2.4 then follows from applying Corollary 2.3 to $h_{k_{0}}$ and using the rank theorem. 


\section{On $k$-approximate formal deformations with $k=1,2$; proofs of Corollaries 2.6-2.8}

In this last section, we discuss the notion of $k$-approximate formal deformations, for $k=$ 1,2 , and provide some existence/non-existence results for such objects in some important circumstances. This will allow us to derive Corollaries 2.6-2.8.

\subsection{1-approximate formal deformations}

We have the following easy characterization of formal CR maps admitting 1-approximate formal deformations.

Proposition 6.1. Let $M \subset \mathbb{C}^{N}$ and $M^{\prime} \subset \mathbb{C}^{N^{\prime}}$ be generic real-analytic submanifolds, with $N, N^{\prime} \geqslant 2$, and let $p \in M$. Let $H:\left(\mathbb{C}_{z}^{N}, p\right) \rightarrow \mathbb{C}_{w}^{N^{\prime}}$ be a formal holomorphic map with $H(M) \subset M^{\prime}$. Then, $H$ admits a 1-approximate formal deformation if and only if there exists a non-zero formal holomorphic vector field

$$
X(z)=\sum_{j=1}^{N^{\prime}} X_{j}(z) \frac{\partial}{\partial w_{j}}, \quad X_{j}(z) \in \mathbb{C}[[z-p]], \quad j=1, \ldots, N^{\prime},
$$

tangent to $M^{\prime}$ along $H(M)$.

Proof. Choose $\varrho=\left(\varrho_{1}, \ldots, \varrho_{d}\right) \in(\mathbb{R}\{w-H(p), \bar{w}-\overline{H(p)}\})^{d}$ such that $M^{\prime}$ is given by the zero-set of $\varrho$ near $H(p)$ with $\partial \varrho_{1} \wedge \ldots \wedge \partial \varrho_{d} \neq 0$ near $H(p)$, where $d=\operatorname{codim}_{\mathbb{R}} M^{\prime}$.

Note that $H$ admits a 1-approximate formal deformation if and only if there exists a non-zero formal holomorphic map $X:\left(\mathbb{C}^{N}, p\right) \rightarrow \mathbb{C}^{N^{\prime}}$ such that

$$
\left.\varrho(H(z)+t X(z), \overline{H(z)+t X(z)})\right|_{z \in M}=O\left(|t|^{2}\right), \quad t \in \mathbb{C} .
$$

Since $H(M) \subset M^{\prime},(6.2)$ is equivalent to say that the formal holomorphic vector field

$$
X(z)=\sum_{j=1}^{N^{\prime}} X_{j}(z) \frac{\partial}{\partial w_{j}}
$$

is tangent to $M^{\prime}$ along $H(M)$. This completes the proof of the proposition.

From Proposition 6.1, we easily get the following.

Proposition 6.2. Let $M$ and $M^{\prime}$ be generic real-analytic submanifolds in $\mathbb{C}^{N}$, with $N \geqslant 2$, and let $p \in M$. Let $H:\left(\mathbb{C}^{N}, p\right) \rightarrow \mathbb{C}^{N}$ be a formal holomorphic map with $H(M) \subset M^{\prime}$ satisfying $\mathrm{Rk} H=N$. Then, $H$ admits a 1-approximate formal deformation if and only if $M$ is holomorphically degenerate at $p$. 
Proof. First recall that $M$ is holomorphically degenerate at $p$ if and only if there exists a non-zero formal holomorphic vector field with coefficients in $\mathbb{C}[[z-p]]$ that is tangent to $M$ (see [BER3] for more details). Let $J_{H}(z)$ be the Jacobian matrix of the formal map $H$ and denote by $C(z)=\left(C_{i j}(z)\right)_{1 \leqslant i, j \leqslant N}$ the classical adjoint of $J_{H}(z)^{T}$. Then, the reader can easily check that, if

$$
X=\sum_{j=1}^{N} X_{j}(z) \frac{\partial}{\partial z_{j}}
$$

is a formal holomorphic vector field at $p$ tangent to $M$, then the formal holomorphic vector field

$$
\widetilde{X}:=X(H)=\sum_{j=1}^{N} X\left(H_{j}\right) \frac{\partial}{\partial w_{j}}
$$

is tangent to $M^{\prime}$ along $H(M)$. Conversely, if

$$
Y=\sum_{j=1}^{N} Y_{j}(z) \frac{\partial}{\partial w_{j}}
$$

is a formal holomorphic vector field at $p$ tangent to $M^{\prime}$ along $H(M)$, then the formal holomorphic vector at $p$

$$
\sum_{j=1}^{N} \sum_{\nu=1}^{N} Y_{\nu}(z) C_{\nu j}(z) \frac{\partial}{\partial z_{j}}
$$

is tangent to $M$.

Using this, the fact that $\operatorname{det} C(z) \not \equiv 0$ ( since $H$ is of $\operatorname{rank} N$ ) and Proposition 6.1, we reach the desired conclusion.

Proposition 6.2 together with Theorem 2.2 then immediately imply Corollary 2.6.

\subsection{2-approximate formal deformations in the Levi-non-degenerate case}

We first start with the following easy fact mentioned in $\S 2.1$. Recall that a generic submanifold in $\mathbb{C}^{N}$ is called strongly pseudoconvex if it is locally contained in a strongly pseudoconvex real hypersurface.

Proposition 6.3. Let $M$ and $M^{\prime}$ be generic real-analytic submanifolds in $\mathbb{C}^{N}$ and $\mathbb{C}^{N^{\prime}}$, respectively, with $N, N^{\prime} \geqslant 2$, and let $p \in M$. Let $H:\left(\mathbb{C}^{N}, p\right) \rightarrow \mathbb{C}^{N}$ be a formal holomorphic map with $H(M) \subset M^{\prime}$. If $M^{\prime}$ is strongly pseudoconvex, then $H$ does not admit any 2-approximate formal deformation. 
Proof. We first note that, if we prove the proposition when $M^{\prime}$ is a hypersurface, then the general case follows by definition. Hence, we assume that $M^{\prime} \subset \mathbb{C}^{N^{\prime}}$ is a strongly pseudoconvex hypersurface passing through $H(p)$ with $H(M) \subset M^{\prime}$. Without loss of generality, we may assume that $p=0$ and $H(p)=0$. We can choose a real-analytic defining function $r(w, \bar{w})$ of $M^{\prime}$ near 0 such that the complex Hessian of $r$ is positive definite on $T_{0}^{c} M^{\prime}$. Let us assume that there is a 2-approximate formal deformation

$$
B:\left(\mathbb{C}^{N} \times \mathbb{C}, 0\right) \longrightarrow\left(\mathbb{C}^{N^{\prime}}, 0\right)
$$

for $H$. This means that we have

$$
\left.r(B(z, t), \overline{B(z, t)})\right|_{z \in M}=O\left(|t|^{3}\right)
$$

Differentiating (6.3) with respect to $t$ and $\bar{t}$ and then evaluating at $t=0$, we get

$$
\left.\partial_{t} B(z, 0) \cdot r_{w \bar{w}}(H(z), \overline{H(z)}) \cdot \overline{\partial_{t} B(z, 0)}\right|_{M}=0 .
$$

From our choice of $r$, equality (6.4) implies that $\partial_{t} B(z, 0)=0$ for $z \in M$ (see [LM1, Lemma 6.1]), and hence that $\partial_{t} B(z, 0)=0$ in $\mathbb{C}[[z]]$, since $M$ is generic. This contradicts the fact that $B$ is an approximate formal deformation. The proof of the proposition is complete.

The situation regarding the existence of 2-approximate formal deformations for CR transversal maps between Levi-non-degenerate hypersurfaces is somewhat more interesting than the strongly pseudoconvex case. In that case, the existence of 2-approximate formal deformations is related to the signature and cosignature of the hypersurfaces. We will prove the following.

Proposition 6.4. Let $M \subset \mathbb{C}^{N}$ and $M^{\prime} \subset \mathbb{C}^{N^{\prime}}$ be (connected) real-analytic Levi-nondegenerate hypersurfaces, of signature $\ell$ and $\ell^{\prime}$, respectively, with $N, N^{\prime} \geqslant 2$. Let $p \in M$ and let $H:(M, p) \rightarrow M^{\prime}$ be a formal holomorphic $C R$ transversal mapping. If $H$ admits a 2-approximate formal deformation, then $M$ and $M^{\prime}$ have different signatures (i.e. $\ell^{\prime} \neq \ell$ ) and different cosignatures (i.e. $N^{\prime}-\ell^{\prime} \neq N-\ell$ ).

Remark 6.5. We remark that Proposition 6.4 is optimal in the following sense. Given $N$ and $N^{\prime}$, and signatures $\ell$ and $\ell^{\prime}$ (satisfying $\ell \leqslant \frac{1}{2}(N-1), \ell^{\prime} \leqslant \frac{1}{2}\left(N^{\prime}-1\right)$ ), we say that $\left(\ell, \ell^{\prime}\right)$ is an admissible pair of signatures if either $\ell^{\prime} \geqslant \ell$ and $N^{\prime}-1-\ell^{\prime} \geqslant N-1-\ell$, or $\ell^{\prime} \geqslant N-1-\ell$. It is well known (see, e.g., $[\mathrm{BH}]$ and $[\mathrm{BEH}]$ ) that formal $\mathrm{CR}$ transversal mappings do not exist if $\left(\ell, \ell^{\prime}\right)$ is not admissible. The following is therefore a converse to Proposition 6.4. For any set of admissible signatures $\left(\ell, \ell^{\prime}\right)$ which satisfy $\ell^{\prime} \neq \ell$ and $N^{\prime}-\ell^{\prime} \neq N-\ell$, there exist explicit examples of germs of real-analytic Levi-non-degenerate 
hypersurfaces $M \subset \mathbb{C}^{N}$ and $M^{\prime} \subset \mathbb{C}^{N^{\prime}}$ with these signatures and a convergent formal $\mathrm{CR}$ transversal map between $M$ and $M^{\prime}$ admitting a 2-approximate formal deformation (in fact, even a holomorphic deformation). Indeed, to see that, one easily checks that, in the above described setting, we necessarily have $\ell^{\prime}>\ell$ and $N^{\prime}-\ell^{\prime}>N-\ell$. Choose now for $M$ the hyperquadric in $\mathbb{C}_{z}^{N}$ with $\ell$ positive eigenvalues, and for $M^{\prime}$ the hyperquadric in $\mathbb{C}_{w}^{N^{\prime}}$ with $\ell^{\prime}$ positive eigenvalues, i.e.

$$
\begin{gathered}
M: \operatorname{Im} z_{n+1}=\|\tilde{z}\|^{2}-\|\hat{z}\|^{2}, \\
M^{\prime}: \operatorname{Im} w_{n^{\prime}+1}=\|\widetilde{w}\|^{2}-\|\widehat{w}\|^{2},
\end{gathered}
$$

where we write $N=n+1, N^{\prime}=n^{\prime}+1, z=\left(\tilde{z}, \hat{z}, z_{n+1}\right) \in \mathbb{C}^{\ell} \times \mathbb{C}^{n-\ell} \times \mathbb{C}, w=\left(\widetilde{w}, \widehat{w}, w_{n^{\prime}+1}\right) \in$ $\mathbb{C}^{\ell^{\prime}} \times \mathbb{C}^{n^{\prime}-\ell^{\prime}} \times \mathbb{C}$ and $\|\tilde{z}\|^{2}=\sum_{1 \leqslant j \leqslant \ell}\left|z_{j}\right|^{2}$, and analogously for the other norm quantities involved. Then, the convergent $\mathrm{CR}$ transversal holomorphic map $w=H(z)$ sending $M$ into $M^{\prime}$ given by $w_{n^{\prime}+1}=z_{n+1}$ and $\widetilde{w}=(\tilde{z}, 0), \widehat{w}=(\hat{z}, 0)$ admits the holomorphic deformation $B(z, t)=\left(\tilde{z}, t, 0, \hat{z}, t, 0, z_{n+1}\right), t \in \mathbb{C}$.

Remark 6.6. As an immediate consequence of Remark 6.5, it follows that the condition on the signatures in Corollary 2.7 is optimal. Indeed, from the previous remark, we get that, for any set of admissible signatures $\left(\ell, \ell^{\prime}\right)$ which satisfy $\ell^{\prime} \neq \ell$ and $N^{\prime}-\ell^{\prime} \neq N-\ell$, there exist explicit examples of germs of real-analytic Levi-non-degenerate hypersurfaces $M \subset \mathbb{C}^{N}$ and $M^{\prime} \subset \mathbb{C}^{N^{\prime}}$ with these signatures and having divergent formal $\mathrm{CR}$ transversal mappings.

Proposition 6.4 will be a consequence of the following result, whose proof is in part inspired by that of [Mir4, Proposition 3.1].

Lemma 6.7. Let $M \subset \mathbb{C}_{z}^{N}$ and $M^{\prime} \subset \mathbb{C}_{w}^{N^{\prime}}$ be real-analytic Levi-non-degenerate hypersurfaces passing through the origin, with $N^{\prime} \geqslant N \geqslant 2$. Write $N=n+1$ and $N^{\prime}=n^{\prime}+1$, and assume that $M$ and $M^{\prime}$ are given by local real-defining functions $\varrho$ and $\varrho^{\prime}$ near the origin as follows:

$$
\begin{gathered}
\varrho(z, \bar{z}):=\operatorname{Im} z_{n+1}+\sum_{j=1}^{e}\left|z_{j}\right|^{2}-\sum_{j=e+1}^{n}\left|z_{j}\right|^{2}+O\left(|z|^{3}\right) \\
\varrho^{\prime}(w, \bar{w}):=\operatorname{Im} w_{n^{\prime}+1}+\sum_{j=1}^{e^{\prime}}\left|w_{j}\right|^{2}-\sum_{j=e^{\prime}+1}^{n^{\prime}}\left|w_{j}\right|^{2}+O\left(|w|^{3}\right),
\end{gathered}
$$

where $1 \leqslant e \leqslant n$ and $1 \leqslant e^{\prime} \leqslant n^{\prime}$. Let $H:(M, 0) \rightarrow\left(M^{\prime}, 0\right)$ be a formal CR transversal holomorphic map such that

$$
\frac{\partial H_{n^{\prime}+1}}{\partial z_{n+1}}(0)>0 .
$$

If $H$ admits a 2-approximate formal deformation, then $0<e^{\prime}-e<N^{\prime}-N$. 
Proof. By [BH, pp. 385-387], we have $N^{\prime} \geqslant N, e^{\prime} \geqslant e, n^{\prime}-e^{\prime} \geqslant n-e$ and there exist local holomorphic coordinates $z^{*}$ in $\mathbb{C}^{n^{\prime}+1}$ such that $M^{\prime}$ is given near the origin in these new coordinates by the vanishing of a real-analytic function $\varrho^{*}\left(z^{*}, \overline{z^{*}}\right)$ of the form

$$
\varrho^{*}\left(z^{*}, \overline{z^{*}}\right)=\operatorname{Im} z_{n^{\prime}+1}^{*}+\sum_{j=1}^{e}\left|z_{j}^{*}\right|^{2}-\sum_{j=e+1}^{n}\left|z_{j}^{*}\right|^{2}+\sum_{j=n+1}^{n+e^{\prime}-e}\left|z_{j}^{*}\right|^{2}-\sum_{j=n+1+e^{\prime}-e}^{n^{\prime}}\left|z_{j}^{*}\right|^{2}+R\left(z^{*}, \overline{z^{*}}\right),
$$

where $R\left(z^{*}, \overline{z^{*}}\right)=O\left(\left|z^{*}\right|^{3}\right)$. Furthermore, in such coordinates, we may write $H=(f, \varphi, g) \in$ $\mathbb{C}^{n} \times \mathbb{C}^{n^{\prime}-n} \times \mathbb{C}$, where the formal map $H$ satisfies the following normalization conditions

$$
(f(z), g(z))=z+O\left(|z|^{2}\right), \quad \varphi(z)=O\left(|z|^{2}\right) .
$$

By assumption, $H$ admits a 2-approximate formal deformation $B:\left(\mathbb{C}^{N} \times \mathbb{C}^{r}, 0\right) \rightarrow\left(\mathbb{C}^{N^{\prime}}, 0\right)$. Without loss of generality, we may assume that $r=1$. We write

$$
B(z, t)=(f(z, t), \varphi(z, t), g(z, t)) \in \mathbb{C}^{n} \times \mathbb{C}^{n^{\prime}-n} \times \mathbb{C},
$$

so that $f(z, 0)=f(z), \varphi(z, 0)=\varphi(z)$ and $g(z, 0)=g(z)$. We also write

$$
f(z, t)=\left(f_{1}(z, t), \ldots, f_{n}(z, t)\right) \quad \text { and } \quad \varphi(z, t)=\left(\varphi_{n+1}(z, t), \ldots, \varphi_{n^{\prime}}(z, t)\right) .
$$

It will be convenient to view the power series maps $B(z, t)$ and $H(z)$ as power series restricted to $M \times \mathbb{C}$. Hence, we may use $x=\left(z_{1}, \ldots, z_{n}, \bar{z}_{1}, \ldots, \bar{z}_{n}, \operatorname{Re} z_{n+1}\right) \in \mathbb{R}^{2 n+1}$ as local coordinates for $M$ near 0 , and write $B(x, t)$ and $H(x)$ for the corresponding restricted power series. We also choose a basis of real-analytic CR vector fields $\left(\bar{L}_{j}\right)_{1 \leqslant j \leqslant n}$ for $M$ near 0 represented in the above chosen $x$ coordinates. In addition, we write $B^{\prime}(x, t)$ for $\partial B / \partial t(x, t)$, as well as for all its components. We define $\widehat{\mathcal{I}}_{H}, \widehat{\mathcal{I}}_{\varphi}$ and $\widehat{\mathcal{I}}_{f, \varphi}$ to be the ideals in $\mathbb{C}[[x]]$ generated by the components of $B^{\prime}(x, 0), \varphi^{\prime}(x, 0)$ and $\left(f^{\prime}(x, 0), \varphi^{\prime}(x, 0)\right)$, respectively. The ideal $\widehat{\mathfrak{m}}$ denotes the usual maximal ideal of $\mathbb{C}[[x]]$.

Since $B$ is 2-approximate deformation, we have the power series identity, in $\mathbb{C}[[x, t, \bar{t}]]$,

$$
\begin{aligned}
\operatorname{Im} g(x, t)+\sum_{j=1}^{e} & \left|f_{j}(x, t)\right|^{2}-\sum_{j=e+1}^{n}\left|f_{j}(x, t)\right|^{2}+\sum_{j=n+1}^{n+e^{\prime}-e}\left|\varphi_{j}(x, t)\right|^{2} \\
& -\sum_{j=n+1+e^{\prime}-e}^{n^{\prime}}\left|\varphi_{j}(x, t)\right|^{2}+R(B(x, t), \overline{B(x, t)})=O\left(|t|^{3}\right) .
\end{aligned}
$$

Differentiating (6.7) with respect to $t$ yields

$$
\begin{aligned}
\frac{1}{2 i} g^{\prime}(x, t)+\sum_{j=1}^{e} f_{j}^{\prime}(x, t) \overline{f_{j}(x, t)}-\sum_{j=e+1}^{n} f_{j}^{\prime}(x, t) \overline{f_{j}(x, t)}+\sum_{j=n+1}^{n+e^{\prime}-e} \varphi_{j}^{\prime}(x, t) \overline{\varphi_{j}(x, t)} \\
-\sum_{j=n+1+e^{\prime}-e}^{n^{\prime}} \varphi_{j}^{\prime}(x, t) \overline{\varphi_{j}(x, t)}+\frac{\partial}{\partial t}\{R(B(x, t), \overline{B(x, t)})\}=O\left(|t|^{2}\right) .
\end{aligned}
$$


Evaluating (6.8) at $t=0$ yields the following identity in $\mathbb{C}[[x]]$ :

$$
\begin{aligned}
\frac{1}{2 i} g^{\prime}(x, 0)+\sum_{j=1}^{e} & f_{j}^{\prime}(x, 0) \overline{f_{j}(x, 0)}-\sum_{j=e+1}^{n} f_{j}^{\prime}(x, 0) \overline{f_{j}(x, 0)}+\sum_{j=n+1}^{n+e^{\prime}-e} \varphi_{j}^{\prime}(x, 0) \overline{\varphi_{j}(x, 0)} \\
& -\sum_{j=n+1+e^{\prime}-e}^{n^{\prime}} \varphi_{j}^{\prime}(x, 0) \overline{\varphi_{j}(x, 0)}+\left.\frac{\partial}{\partial t}\{R(B(x, t), \overline{B(x, t)})\}\right|_{t=0}=0 .
\end{aligned}
$$

Since $R\left(z^{*}, \overline{z^{*}}\right)=O\left(\left|z^{*}\right|^{3}\right)$,

$$
\left.\frac{\partial}{\partial t}\{R(B(x, t), \overline{B(x, t)})\}\right|_{t=0}
$$

clearly belongs to the product ideal $\widehat{\mathfrak{m}} \widehat{\mathcal{I}}_{H}$. Hence, from $(6.9)$, we conclude that $g^{\prime}(x, 0)$ belongs to the ideal $\widehat{\mathfrak{m}} \widehat{\mathcal{I}}_{f, \varphi}$, and thus $\widehat{\mathcal{I}}_{H}=\widehat{\mathcal{I}}_{f, \varphi}$. Next, applying the CR vector fields $\bar{L}_{k}$ to $(6.8)$ for $k=1, \ldots, n$, and evaluating at $t=0$, we obtain

$$
\begin{aligned}
& \sum_{j=1}^{e} f_{j}^{\prime}(x, 0) \bar{L}_{k} \bar{f}_{j}(x, 0)-\sum_{j=e+1}^{n} f_{j}^{\prime}(x, 0) \bar{L}_{k} \bar{f}_{j}(x, 0)+\sum_{j=n+1}^{n+e^{\prime}-e} \varphi_{j}^{\prime}(x, 0) \bar{L}_{k} \bar{\varphi}_{j}(x, 0) \\
&-\sum_{j=n+1+e^{\prime}-e}^{n^{\prime}} \varphi_{j}^{\prime}(x, 0) \bar{L}_{k} \bar{\varphi}_{j}(x, 0)+\left.\bar{L}_{k} \frac{\partial}{\partial t}\{R(B(x, t), \overline{B(x, t)})\}\right|_{t=0}=0 .
\end{aligned}
$$

Again, since $R\left(z^{*}, \bar{z}^{*}\right)=O\left(\left|z^{*}\right|^{3}\right)$, the last term of (6.10) belongs to the ideal $\widehat{\mathfrak{m}} \widehat{\mathcal{I}}_{H}=\widehat{\mathfrak{m}} \widehat{\mathcal{I}}_{f, \varphi}$. Using the fact $\varphi(z)=O\left(|z|^{2}\right)$ from (6.6), we conclude from (6.10) that, for $k=1, \ldots, n$,

$$
\sum_{j=1}^{e} f_{j}^{\prime}(x, 0) \bar{L}_{k} \bar{f}_{j}(x, 0)-\sum_{j=e+1}^{n} f_{j}^{\prime}(x, 0) \bar{L}_{k} \bar{f}_{j}(x, 0) \in \widehat{\mathfrak{m}} \widehat{\mathcal{I}}_{f, \varphi} .
$$

We recall that

$$
(f(z), g(z))=z+O\left(|z|^{2}\right)
$$

by (6.6), which implies that the matrix $\left(\bar{L}_{k} \bar{f}_{j}(0)\right)_{j, k}$ is invertible, and therefore, (6.11) shows that $f_{j}^{\prime}(x, 0) \in \widehat{\mathfrak{m}} \widehat{\mathcal{I}}_{\varphi}$ for $j=1, \ldots, n$. Note that this latter statement also implies that $g^{\prime}(x, 0) \in \widehat{\mathfrak{m}} \widehat{\mathcal{I}}_{\varphi}$, since we already know that $g^{\prime}(x, 0) \in \widehat{\mathfrak{m}} \widehat{\mathcal{I}}_{f, \varphi}$.

Differentiating (6.8) with respect to $\bar{t}$ and evaluating at $t=0$, we get

$$
\begin{aligned}
\sum_{j=1}^{e}\left|f_{j}^{\prime}(x, 0)\right|^{2} & -\sum_{j=e+1}^{n}\left|f_{j}^{\prime}(x, 0)\right|^{2}+\sum_{j=n+1}^{n+e^{\prime}-e}\left|\varphi_{j}^{\prime}(x, 0)\right|^{2} \\
& -\sum_{j=n+1+e^{\prime}-e}^{n^{\prime}}\left|\varphi_{j}^{\prime}(x, 0)\right|^{2}+\left.\frac{\partial}{\partial \bar{t} \partial t}\{R(B(x, t), \overline{B(x, t)})\}\right|_{t=0}=0 .
\end{aligned}
$$


Since each $f_{j}^{\prime}(x, 0)$ belongs to $\widehat{\mathfrak{m}} \widehat{\mathcal{I}}_{\varphi}$, we see that we may write

$$
\left.\frac{\partial}{\partial \bar{t} \partial t}\{R(B(x, t), \overline{B(x, t)})\}\right|_{t=0}=\sum_{j, k} A_{j k}(x) \varphi_{j}^{\prime}(x, 0) \overline{\varphi_{k}^{\prime}(x, 0)}
$$

where each $A_{j k}(x) \in \widehat{\mathfrak{m}}$. Recall now that, since $B$ is an approximate formal deformation, we necessarily have $B^{\prime}(x, 0) \not \equiv 0$. As each $f_{j}^{\prime}(x, 0)$ and $g^{\prime}(x, 0)$ belong to $\widehat{\mathfrak{m}} \widehat{\mathcal{I}}_{\varphi}$, there exists $m \in\left\{n+1, \ldots, n^{\prime}\right\}$ such that $\varphi_{m}^{\prime}(x, 0) \not \equiv 0$. There exists therefore $0 \leqslant \ell<+\infty$ such that, for each $n+1 \leqslant j \leqslant n^{\prime}$, one may write the homogeneous expansion $\varphi_{j}^{\prime}(x, 0)=\sum_{\nu \geqslant \ell} \theta_{j, \nu}(x)$, where $\theta_{j, \ell}(x) \not \equiv 0$ for some $j$. Comparing the homogeneous terms of degree $2 \ell$ in $(6.12)$ and using (6.13), we obtain

$$
\sum_{j=n+1}^{n+e^{\prime}-e}\left|\theta_{j, \ell}(x)\right|^{2}-\sum_{j=n+1+e^{\prime}-e}^{n^{\prime}}\left|\theta_{j, \ell}(x)\right|^{2}=0 .
$$

Equality (6.14) implies that we necessarily have $e^{\prime}-e>0$ and $e^{\prime}-e<n^{\prime}-n=N^{\prime}-N$. The proof of Lemma 6.7 is complete.

Proof of Proposition 6.4. We may assume that $p=0$ and $H(p)=0$. One may find local holomorphic coordinates $z \in \mathbb{C}^{N}$ and $z^{\prime} \in \mathbb{C}^{N^{\prime}}$ such that the germs at 0 of $M$ and $M^{\prime}$ are respectively given by the vanishing of real-analytic functions $\varrho$ and $\varrho^{\prime}$ as given by (6.5), with $e=\ell$ and $e^{\prime}=\ell^{\prime}$. As is well known, $\partial H_{n^{\prime}+1} / \partial z_{n+1}$ is real-valued (see, e.g., $[\mathrm{BH}])$. We have to distinguish the following two situations.

- $\partial H_{n^{\prime}+1} / \partial z_{n+1}>0$. Then, it follows from Lemma 6.7 that $\ell^{\prime}-\ell \notin\left\{0, N^{\prime}-N\right\}$.

- $\partial H_{n^{\prime}+1} / \partial z_{n+1}<0$. Then, by making the new change of coordinates

$$
z \longmapsto\left(z_{1}, \ldots, z_{n},-z_{n+1}\right)
$$

we are back to the situation where we can apply Lemma 6.7 with $e=N-1-\ell$ and $e^{\prime}=\ell^{\prime}$. Hence, in this setting, we get that $0<\ell^{\prime}-(N-1-\ell)<N^{\prime}-N$. We leave it to the reader to check that these last inequalities prevent $\ell^{\prime}-\ell$ to be equal to 0 and $N^{\prime}-N$, as the signatures $\ell$ and $\ell^{\prime}$ must satisfy the inequalites $\ell \leqslant \frac{1}{2}(N-1)$ and $\ell^{\prime} \leqslant \frac{1}{2}\left(N^{\prime}-1\right)$. The proof of Proposition 6.4 is therefore complete.

Corollary 2.7 now follows immediately from Theorem 2.2 and Proposition 6.4.

\subsection{2-approximate formal deformations into the tube over the light cone}

The following result describes precisely the formal maps valued into the tube over the light cone that admit a 2-approximate formal deformation. 
Proposition 6.8. Let $M \subset \mathbb{C}^{N}$ be a generic real-analytic submanifold in $\mathbb{C}^{N}$, of finite type at $p \in M$, and $\mathbb{T}^{N^{\prime}} \subset \mathbb{C}^{N^{\prime}}$ be the tube over the light cone given by (2.1), with $N, N^{\prime} \geqslant 2$. If the formal holomorphic map $H:(M, p) \rightarrow \mathbb{T}^{N^{\prime}}$ admits a 2-approximate formal deformation, then there exist a formal holomorphic power series $\mu(z) \in \mathbb{C}[[z-p]]$ and $\alpha_{j}, \eta_{j} \in \mathbb{R}, 1 \leqslant j \leqslant N^{\prime}-1$, with $\sum_{j=1}^{N^{\prime}-1} \alpha_{j}^{2}=1$, such that

$$
H(z)=\left(\alpha_{1} \mu(z)+i \eta_{1}, \ldots, \alpha_{N^{\prime}-1} \mu(z)+i \eta_{N^{\prime}-1}, \mu(z)\right) .
$$

We note that Proposition 6.8 immediately implies, together with Theorem 2.2 the following result.

COROLlary 6.9. Let $M \subset \mathbb{C}^{N}$ be a generic real-analytic submanifold in $\mathbb{C}^{N}$, of finite type at $p \in M$, and $\mathbb{T}^{N^{\prime}} \subset \mathbb{C}^{N^{\prime}}$ be the tube over the light cone, with $N, N^{\prime} \geqslant 2$. Then any divergent formal holomorphic map $H:(M, p) \rightarrow \mathbb{T}^{N^{\prime}}$ is of the form (6.15), for some divergent formal power series $\mu$.

From Corollary 6.9, we immediately get Corollary 2.8. The proof of Proposition 6.8 is mainly an adaptation of the arguments of the proof of [Mir4, Lemma 2.3].

Proof of Proposition 6.8. Let $B(z, t)$ be a 2-approximate formal deformation for $\left(M, M^{\prime}, H\right)$, with $t \in \mathbb{C}^{r}$. Without loss of generality, we may assume that $r=1$. We write

$$
B(z, t)=\left(B^{1}(z, t), \ldots, B^{N^{\prime}}(z, t)\right), \quad B^{\nu}(z, t)=\sum_{j=0}^{2} B_{j}^{\nu}(z) t^{j}, \quad \nu=1, \ldots, N^{\prime}
$$

We have

$$
\left(\operatorname{Re} B^{N^{\prime}}(z, t)\right)^{2}=\sum_{k=1}^{N^{\prime}-1}\left(\operatorname{Re} B^{k}(z, t)\right)^{2}+O\left(|t|^{3}\right), \quad z \in M .
$$

The zero-, first- and second-order terms with respect to $(t, \bar{t})$ in (6.16) give the following system of equations, valid for $z \in M$ :

$$
\left\{\begin{array}{l}
\left(\operatorname{Re} B_{0}^{N^{\prime}}(z)\right)^{2}=\sum_{k=1}^{N^{\prime}-1}\left(\operatorname{Re} B_{0}^{k}(z)\right)^{2} \\
\left(\operatorname{Re} B_{0}^{N^{\prime}}\right) B_{1}^{N^{\prime}}(z)=\sum_{k=1}^{N^{\prime}-1}\left(\operatorname{Re} B_{0}^{k}(z)\right) B_{1}^{k}(z), \\
\left|B_{1}^{N^{\prime}}(z)\right|^{2}=\sum_{k=1}^{N^{\prime}-1}\left|B_{1}^{k}(z)\right|^{2}
\end{array}\right.
$$

Since $B(z, t)$ is a formal deformation, in view of the last equation of (6.17), we must have $B_{1}^{N^{\prime}}(z) \not \equiv 0$ for $z \in M$. Furthermore, we may assume that

$$
\left.\operatorname{Re} B_{0}^{N^{\prime}}(z)\right|_{z \in M} \not \equiv 0
$$


Indeed, if not, then we see from the first equation of (6.17) that this implies that the restriction to $M$ of every formal holomorphic power series $B_{0}^{\nu}(z)$ is purely imaginary. Since $M$ is of finite type, by [JLM], each $B_{0}^{\nu}(z), \nu=1, \ldots, N^{\prime}$, must be a purely imaginary constant. Then, it is not difficult to see that the map $H$ is of the form (6.15), since $H=\left(B_{0}^{1}, \ldots, B_{0}^{N^{\prime}}\right)$. Hence, in what follows we may assume that (6.18) holds.

With this assumption, we can consider the set of equations

$$
1=\sum_{k=1}^{N^{\prime}-1} \frac{\left(\operatorname{Re} B_{0}^{k}(z)\right)^{2}}{\left(\operatorname{Re} B_{0}^{N^{\prime}}(z)\right)^{2}}, \quad 1=\sum_{k=1}^{N^{\prime}-1} \frac{\left(\operatorname{Re} B_{0}^{k}(z)\right)}{\left(\operatorname{Re} B_{0}^{N^{\prime}}\right)} \frac{B_{1}^{k}(z)}{B_{1}^{N^{\prime}}(z)}, \quad 1=\sum_{k=1}^{N^{\prime}-1} \frac{B_{1}^{k}(z)}{B_{1}^{N^{\prime}}(z)} \frac{\overline{B_{1}^{k}(z)}}{B_{1}^{N^{\prime}}(z)}
$$

in $\mathbb{C}((M))$. It follows that

$$
\sum_{k=1}^{N^{\prime}-1}\left(\frac{\left(\operatorname{Re} B_{0}^{k}(z)\right)}{\left(\operatorname{Re} B_{0}^{N^{\prime}}(z)\right)}-\frac{B_{1}^{k}(z)}{B_{1}^{N^{\prime}}(z)}\right) \overline{\left(\frac{\left(\operatorname{Re} B_{0}^{k}(z)\right)}{\left(\operatorname{Re} B_{0}^{N^{\prime}}(z)\right)}-\frac{B_{1}^{k}(z)}{B_{1}^{N^{\prime}}(z)}\right)}=0 \quad \text { in } \mathbb{C}((M)) .
$$

In other words, the power series

$$
\varphi^{k}(z, \bar{z})=\left(\operatorname{Re} B_{0}^{k}(z)\right) B_{1}^{N^{\prime}}(z) \overline{B_{1}^{N^{\prime}}(z)}-B_{1}^{k}(z) \overline{B_{1}^{N^{\prime}}(z)}\left(\operatorname{Re} B_{0}^{N^{\prime}}(z)\right) \in \mathbb{C}[[M]]
$$

for $k=1, \ldots, N^{\prime}-1$, satisfy $\sum_{k=1}^{N^{\prime}-1}\left|\varphi^{k}(z, \bar{z})\right|^{2}=0$, and hence $\varphi^{k}(z, \bar{z})=0$, for $k=1, \ldots, N^{\prime}-1$ and $z \in M$. It follows that

$$
\left(\frac{\operatorname{Re} B_{0}^{1}}{\operatorname{Re} B_{0}^{N^{\prime}}}, \ldots, \frac{\operatorname{Re} B_{0}^{N^{\prime}-1}}{\operatorname{Re} B_{0}^{N^{\prime}}}\right)=\left(\frac{B_{1}^{1}}{B_{1}^{N^{\prime}}}, \ldots, \frac{B_{1}^{N^{\prime}-1}}{B_{1}^{N^{\prime}}}\right) .
$$

Hence, each of the ratios $B_{1}^{\nu}(z) / B_{1}^{N^{\prime}}(z)$, for $\nu=1, \ldots, N^{\prime}-1$, is real-valued when restricted to $M$. By [JLM], the latter ratios must be real constants, that we denote by $\alpha_{\nu}$, satisfying, in view of the last equation of $(6.17), \sum_{1 \leqslant \nu \leqslant N^{\prime}-1} \alpha_{\nu}^{2}=1$. Furthermore, this also yields that, for $\nu=1, \ldots, N^{\prime}-1$,

$$
\left.\operatorname{Re}\left(B_{0}^{\nu}(z)-\alpha_{\nu} B_{0}^{N^{\prime}}(z)\right)\right|_{z \in M}=0 .
$$

Again by [JLM], this implies that there exist $\eta_{\nu} \in \mathbb{R}, \nu=1, \ldots, N^{\prime}-1$, such that

$$
B_{0}^{\nu}(z)-\alpha_{\nu} B_{0}^{N^{\prime}}(z)=i \eta_{\nu}
$$

Noticing that $H=\left(B_{0}^{1}, \ldots, B_{0}^{N^{\prime}}\right)$ and setting $\mu(z):=B_{0}^{N^{\prime}}(z)$, we have reached the desired conclusion.

\section{Acknowledgements}

The authors would like to thank Guillaume Rond for many useful discussions related to $\S 5.1$ of the present paper and for providing the proof of Theorem 5.2. 


\section{References}

[A] Artin, M., On the solutions of analytic equations. Invent. Math., 5 (1968), 277-291.

[BEH] Baouendi, M.S., Ebenfelt, P. \& Huang, X., Holomorphic mappings between hyperquadrics with small signature difference. Amer. J. Math., 133 (2011), 16331661.

[Ber1] Baouendi, M.S., Ebenfelt, P. \& Rothschild, L.P., Parametrization of local biholomorphisms of real analytic hypersurfaces. Asian J. Math., 1 (1997), 1-16.

[BER2] - Real Submanifolds in Complex Space and their Mappings. Princeton Mathematical Series, 47. Princeton Univ. Press, Princeton, NJ, 1999.

[BER3] - Convergence and finite determination of formal CR mappings. J. Amer. Math. Soc., 13 (2000), 697-723.

[BH] Baouendi, M. S. \& Huang, X., Super-rigidity for holomorphic mappings between hyperquadrics with positive signature. J. Differential Geom., 69 (2005), 379-398.

[BMR] Baouendi, M.S., Mir, N. \& Rothschild, L.P., Reflection ideals and mappings between generic submanifolds in complex space. J. Geom. Anal., 12 (2002), 543580 .

[BCH] Berhanu, S., Cordaro, P. D. \& Hounie, J., An Introduction to Involutive Structures. New Mathematical Monographs, 6. Cambridge Univ. Press, Cambridge, 2008.

[BX1] Berhanu, S. \& XIAO, M., On the $C^{\infty}$ version of the reflection principle for mappings between CR manifolds. Amer. J. Math., 137 (2015), 1365-1400.

[BX2] - On the regularity of CR mappings between CR manifolds of hypersurface type. Trans. Amer. Math. Soc., 369 (2017), 6073-6086.

[BG] Bloom, T. \& Graham, I., On "type" conditions for generic real submanifolds of $\mathbb{C}^{n}$. Invent. Math., 40 (1977), 217-243.

[CM] Chern, S. S. \& Moser, J. K., Real hypersurfaces in complex manifolds. Acta Math., 133 (1974), 219-271. Erratum in Acta Math., 150 (1983), 297.

[D1] D'Angelo, J. P., Real hypersurfaces, orders of contact, and applications. Ann. of Math., 115 (1982), 615-637.

[D2] - Finite type and the intersection of real and complex subvarieties, in Several Complex Variables and Complex Geometry, Part 3 (Santa Cruz, CA, 1989), Proc. Sympos. Pure Math., 52, pp. 103-117. Amer. Math. Soc., Providence, RI, 1991.

[D3] - Several Complex Variables and the Geometry of Real Hypersurfaces. Studies in Advanced Mathematics. CRC Press, Boca Raton, FL, 1993.

[DL] Denef, J. \& Lipshitz, L., Ultraproducts and approximation in local rings. II. Math. Ann., 253 (1980), 1-28.

[Fre] Freeman, M., Local complex foliation of real submanifolds. Math. Ann., 209 (1974), $1-30$.

[Fri] Frisch, J., Points de platitude d'un morphisme d'espaces analytiques complexes. Invent. Math., 4 (1967), 118-138.

[GS] Gong, X. \& Stolovitch, L., Real submanifolds of maximum complex tangent space at a CR singular point, I. Invent. Math., 206 (2016), 293-377.

[H] Hauser, H., The classical Artin approximation theorems. Bull. Amer. Math. Soc., 54 (2017), 595-633.

[HR] Hickel, M. \& Rond, G., Approximation of holomorphic solutions of a system of real analytic equations. Canad. Math. Bull., 55 (2012), 752-761.

[HY1] HuAnG, X. \& YIN, W., A Bishop surface with a vanishing Bishop invariant. Invent. Math., 176 (2009), 461-520. 
[HY2] - A codimension two CR singular submanifold that is formally equivalent to a symmetric quadric. Int. Math. Res. Not., (2009), 2789-2828.

[HY3] - Flattening of CR singular points and analyticity of the local hull of holomorphy II. Adv. Math., 308 (2017), 1009-1073.

[JLM] Juhlin, R., LAmel, B. \& Meylan, F., Formal meromorphic functions on manifolds of finite type. Pure Appl. Math. Q., 6 (2010), 1027-1033.

[KS] Kossovskiy, I. \& Shafikov, R., Divergent CR-equivalences and meromorphic differential equations. J. Eur. Math. Soc. (JEMS), 18 (2016), 2785-2819.

[L] LAmel, B., Holomorphic maps of real submanifolds in complex spaces of different dimensions. Pacific J. Math., 201 (2001), 357-387.

[LM1] LAmel, B. \& MiR, N., Convergence of formal CR mappings into strongly pseudoconvex Cauchy-Riemann manifolds. Invent. Math., 210 (2017), 963-985.

[LM2] - On the $\mathcal{C}^{\infty}$ regularity of CR mappings of positive codimension. Adv. Math., 335 (2018), 696-734.

[MMZ1] Meylan, F., Mir, N. \& Zaitsev, D., Approximation and convergence of formal CR-mappings. Int. Math. Res. Not., 4 (2003), 211-242.

[MMZ2] - On some rigidity properties of mappings between CR-submanifolds in complex space, in Journées "Équations aux Dérivées Partielles", Exp. No. XII, 20 pp. Univ. Nantes, Nantes, 2003.

[Mil] Milman, P.D., Complex analytic and formal solutions of real analytic equations in $\mathbb{C}^{n}$. Math. Ann., 233 (1978), 1-7.

[Mir1] MiR, N., Formal biholomorphic maps of real analytic hypersurfaces. Math. Res. Lett., 7 (2000), 343-359.

[Mir2] - Convergence of formal embeddings between real-analytic hypersurfaces in codimension one. J. Differential Geom., 62 (2002), 163-173.

[Mir3] - Artin's approximation theorems and Cauchy-Riemann geometry. Methods Appl. Anal., 21 (2014), 481-502.

[Mir4] - Holomorphic deformations of real-analytic CR maps and analytic regularity of CR mappings. J. Geom. Anal., 27 (2017), 1920-1939.

[Ron] Rond, G., Artin approximation. J. Singul., 17 (2018), 108-192.

[Rot] Rothschild, L. P., Mappings between real submanifolds in complex space, in Explorations in Complex and Riemannian Geometry, Contemp. Math., 332, pp. 253-266. Amer. Math. Soc., Providence, RI, 2003.

[Sta] Stanton, N.K., Infinitesimal CR automorphisms of real hypersurfaces. Amer. J. Math., 118 (1996), 209-233.

[Sto] Stolovitch, L., Family of intersecting totally real manifolds of $\left(\mathbb{C}^{n}, 0\right)$ and germs of holomorphic diffeomorphisms. Bull. Soc. Math. France, 143 (2015), 247-263.

[Su] SunYÉ, J. C., On formal maps between generic submanifolds in complex space. $J$. Geom. Anal., 19 (2009), 944-962.

[W] WaVRIK, J. J., A theorem on solutions of analytic equations with applications to deformations of complex structures. Math. Ann., 216 (1975), 127-142.

[Z] Zaitsev, D., Formal and finite order equivalences. Math. Z., 269 (2011), 687-696. 
BERnhaRd LAMEL

Universität Wien

Fakultät für Mathematik

Oskar-Morgenstern-Platz 1

AT-1090 Vienna

Austria

bernhard. lamel@univie.ac . at

Received September 26, 2017
Nordine Mir

Texas A\&M University at Qatar

Science program

PO Box 23874

Education City, Doha

Qatar

nordine.mir@qatar.tamu .edu 